\title{
REVISED JSim, an open-source modeling system for data analysis
}

\section{[version 2; peer review: 2 approved]}

\author{
Erik Butterworth, Bartholomew E. Jardine, Gary M. Raymond, Maxwell L. Neal, \\ James B. Bassingthwaighte (ii)
}

Dept. of Bioengineering, University of Washington, Seattle, WA 98195, USA

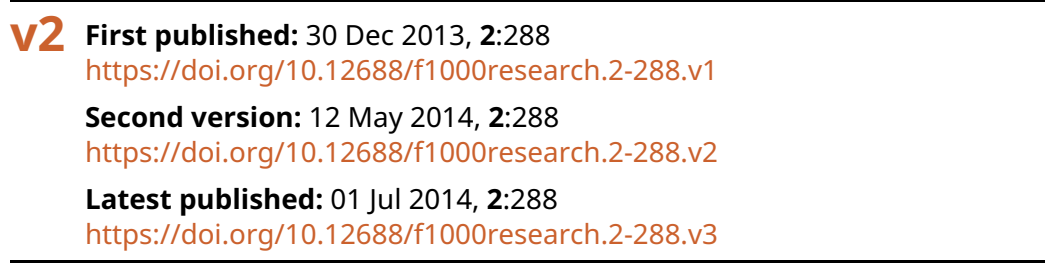

\section{Abstract}

JSim is a simulation system for developing models, designing experiments, and evaluating hypotheses on physiological and pharmacological systems through the testing of model solutions against data. It is designed for interactive, iterative manipulation of the model code, handling of multiple data sets and parameter sets, and for making comparisons among different models running simultaneously or separately. Interactive use is supported by a large collection of graphical user interfaces for model writing and compilation diagnostics, defining input functions, model runs, selection of algorithms solving ordinary and partial differential equations, run-time multidimensional graphics, parameter optimization (8 methods), sensitivity analysis, and Monte Carlo simulation for defining confidence ranges. JSim uses Mathematical Modeling Language (MML) a declarative syntax specifying algebraic and differential equations. Imperative constructs written in other languages (MATLAB, FORTRAN, $\mathrm{C}++$, etc.) are accessed through procedure calls. MML syntax is simple, basically defining the parameters and variables, then writing the equations in a straightforward, easily read and understood mathematical form. This makes JSim good for teaching modeling as well as for model analysis for research. For high throughput applications, JSim can be run as a batch job. JSim can automatically translate models from the repositories for Systems Biology Markup Language (SBML) and CelIML models. Stochastic modeling is supported. MML supports assigning physical units to constants and variables and automates checking dimensional balance as the first step in verification testing. Automatic unit scaling follows, e.g. seconds to minutes, if needed. The JSim Project File sets a standard for reproducible modeling analysis: it includes in one file everything for analyzing a set of experiments: the data, the models, the data fitting, and evaluation of parameter confidence ranges. JSim is open source; it and about 400 human readable open source physiological/biophysical models are available

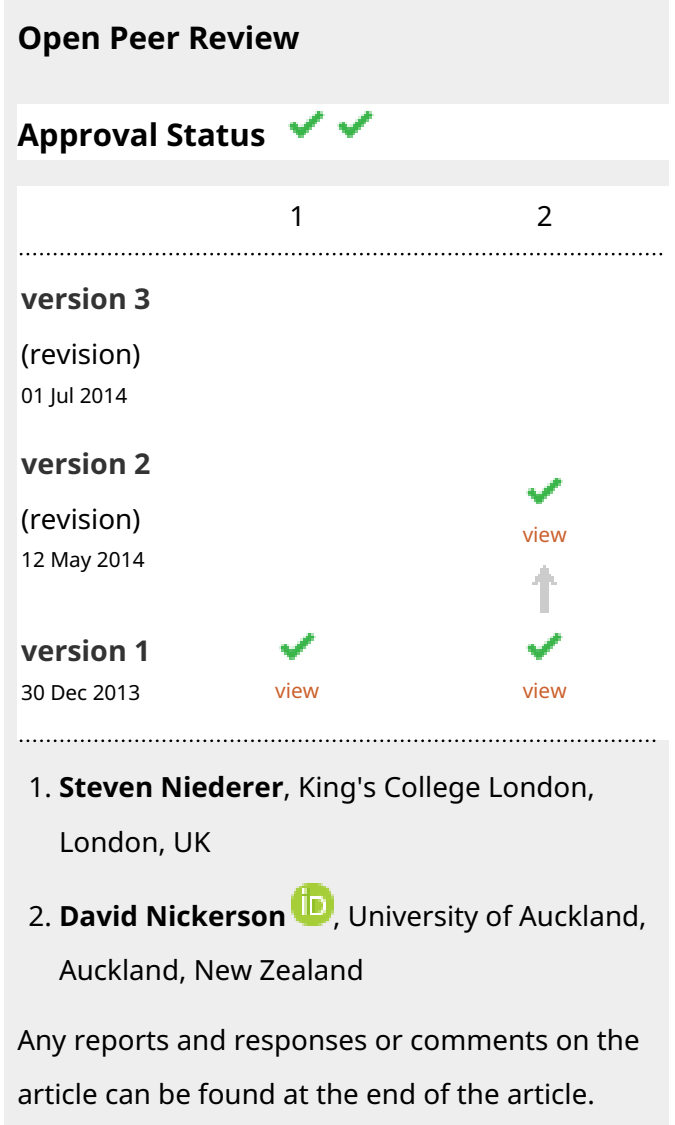


at http://www.physiome.org/jsim/.

\section{Keywords}

experimental data analysis, partial differential and ordinary

differential equations, differential algebraic equations (DAE), JSim,

modeling, optimization, residuals, Monte Carlo, continuous systems,

stochastic systems simulation, sensitivity, time series, reproducible

research, SBML, CelIML.

Corresponding author: James B. Bassingthwaighte (jbb2@u.washington.edu)

Competing interests: No competing interests were disclosed.

Grant information: The development of JSim has been supported by NIH grants HL9719 (PI: JBB), RR1243 (JBB), EB1273 (JBB), HL073598

(PI: R. Corley), EB8407(JBB), GM094503 (PI: D. Beard), GM081070 (PI: H. Sauro) and NSF grant 0506477(JBB).

The funders had no role in study design, data collection and analysis, decision to publish, or preparation of the manuscript.

Copyright: ( $) 2014$ Butterworth E et al. This is an open access article distributed under the terms of the Creative Commons Attribution License, which permits unrestricted use, distribution, and reproduction in any medium, provided the original work is properly cited. Data associated with the article are available under the terms of the Creative Commons Zero "No rights reserved" data waiver (CC0 1.0 Public domain dedication).

How to cite this article: Butterworth E, Jardine BE, Raymond GM et al. JSim, an open-source modeling system for data analysis [version 2; peer review: 2 approved] F1000Research 2014, 2:288 https://doi.org/10.12688/f1000research.2-288.v2

First published: 30 Dec 2013, 2:288 https://doi.org/10.12688/f1000research.2-288.v1 


\section{REVISED Amendments from Version 1}

We have added several new sections in response to reviewer's comments: "Some Alternative Simulation Platforms", "SED-ML Support" (in section "Future Plans"), "Code Verification" and "Using archived models for analyzing one's own data". Two new tables were added to the "Run-Time Performance" section. Furthermore, we have added clarifying wording in various places and 9 new references were included.

See referee reports

\section{Introduction}

The modeling of biological processes starts with defining the hypothesis to be tested in an experiment. To make scientific progress, Platt (Platt, 1964) emphasized defining at least two distinct hypotheses and then designing an experiment with the power to clearly distinguish between these hypotheses. By so doing, at least one of the hypotheses must then be rejected; the rejection marks a stepping-stone in science. If a hypothesis is not rejected then it remains as a potential working hypothesis, the target of further experimentation that eventually will lead to its rejection or improvement.

The virtue of the mathematically-defined hypothesis is that it is clear and precise, and therefore susceptible to contradiction. Arguably, one should use mathematical "in numero experimentation" to define the critical laboratory experiment. Given that the experiment tests whether or not the working hypothesis is compatible with experimental data, then failure to fit the data within a defined level of goodness of fit leads to skepticism about the accuracy of the data or more often, about the structure of the model and leads to its modification or to its outright rejection. Revision of the conjecture follows: science is advanced.

The hypothesis testing cycle is an iterative procedure: design hypothesis (and alternative hypotheses) $\rightarrow$ execute experiment $\rightarrow$ evaluate goodness of fit of model to data $\rightarrow$ either reject the hypothesis and restart, or, alternatively, $\rightarrow$ accept the model as the current working hypothesis and assess the parameters for the specific situation. The working model serves as the current belief until deeper thinking leads to an alternative hypothesis and one restarts the cycle. This philosophical and procedural point of view, more or less guaranteed to make efficient progress in the field, creates definable results step by step, and gives investigators a sense of satisfactory success.

As in physics, models are posed in order to gain deeper understanding and to make predictions. The more realistic the model, the more accurate the prediction. Cause-and-effect models of biological systems are usually deterministic; they are fundamentally different from observationally-based probabilistic associations. The desire is to represent sequences of operations within a dynamic system leading to, and explaining, the observed data (Coatrieux \& Bassingthwaighte, 2006; Bassingthwaighte et al.. 2006a). Standard statistical methods are not central to deciding whether or not to reject the hypothesis, but are indeed helpful in assessing goodness of fit, estimating confidence ranges and co-variances among parameters, and in guiding the investigator in identifying errors or in finding ways to simplify the model.
Over the years we have developed sets of tools to serve these processes. In this article we describe the features of a simulation analysis system, JSim; it is the product of evolutionary improvements in the hypothesis testing cycle. The central goals are to facilitate attempts to fit models to data, and to support the efficient development of computational models that describe and explain the behavior of biological systems (Bassingthwaighte \& Goresky, 1984; Bassingthwaighte et al., 2005; Beard et al., 2005).

Our perspective is embedded in JSim: it is an open-source simulation analysis platform, freely downloadable, running on Linux, Macintosh, and Windows, providing tools for the steps in the modeling analysis of data. There is a naturally occurring sequence of steps to take when one starts with an unanalyzed data set and has the goal of modeling the cause and effect relationships. We have found it useful to follow a simplified summary: The THIRTEEN STEPS:

\section{The THIRTEEN STEPS in the modeling process}

These are proposed as a guide. The ordering is not rigid, but it is wise to cover all of the steps in one's mind when starting and again when finishing up a study. Using the steps in the order listed here almost always works well.

(1) When starting with existing experimental data, plot and display the data so that one can rapidly review and compare multiple data sets. This also prepares for comparing with later model results.

(2) Develop the model, the mathematical formulation of the hypothesis. One may start with one or more existing models or modules of a similar nature (retrieved from a model repository or archival format) and modify it. Construct illustrations of model structure to aid the conceptual approach.

(3) Verify unitary balance in the model equations, an easy first check for model self-consistency.

(4) Select appropriate methods for solving model equations (e.g. differential equation solvers).

(5) Display model solutions graphically and in text listings. Inspect.

(6) Verify the mathematical accuracy of solutions. Check that results are not dependent on temporal or spatial step sizes, that mass or charge is appropriately conserved, and that limiting cases match analytical solutions.

(7) Explore model behavior over wide ranges of parameter values in state-space. (We think of "state space" as being the $\mathrm{N}$-dimensional space enclosing the ranges of values of all of the parameters within which the model is correct numerically and sensible scientifically).

(8) Perform sensitivity analyses, examining the fractional change in model solutions with fractional change in each parameter.

(9) Adjust parameters to fit model solution to data, manually or using an optimizer. Start from different places in parameter space and vary the optimization method to test solution uniqueness.

(10) Assess goodness of model fit to data. Plot residual differences to expose systematic biases.

(11) Examine parameter correlations to identify highly correlated parameters and reduce the number of free parameters in optimizations. Reoptimize. 
(12) Evaluate parameter confidence ranges. The sensitivities at the "best fit", expressed as the local curvature of the optimization cost function give a practical estimate. This can be refined using a Monte Carlo evaluation of parameter likelihoods as probability density functions.

(13) Preserve the source code, multiple data sets, multiple analyses and parameter sets, the settings (for initial and boundary conditions, parameter scans, displays, solver choices, optimizers, Monte Carlo, etc.), the graphs of results, the investigator's notes and descriptions of procedures, plots, etc., all in a single, reproducible, exportable package. Share this package openly with collaborators, reviewers, and the public, a moral and perhaps ethical requirement when the support comes from public funds.

\section{Interpretation of analyses}

What one wants primarily from modeling analysis is insight into mechanisms. JSim is efficient for model development and testing. The fitting of experimental data by model solutions does not provide proof that the model is correct. It says merely that the model can serve as a descriptor under limited range of circumstances, namely those examined in the experimentation. Validity is never provable. Likewise, causation may be identified, but deeper levels may exist to be revealed later.

What does the model predict? Every model, with a little ingenuity, can be queried. What would be the responses to different inputs? How would the system respond if a component were missing or damaged? Predictions then form the basis for the design of the next experimental test. Correct predictions, failing to invalidate the model, do strengthen the confidence in the model but only to the degree commensurate with the comprehensiveness of the particular prediction.

\section{Background}

JSim is the latest in a series of modeling/data analysis programs dating back to SimCon (Knopp et al., 1970) (named for Simulation Control). SimCon provided a text and graphics interface to models written in Fortran. Between 1967 and 1993, the basic methods of data analysis (e.g. function generators, loops, sensitivity, optimization) were developed and refined within the SimCon framework. In 1993, SimCon was replaced by XSim (King et al., 1995), which implemented the same functionality under X-Windows on several Unix-like operating systems (SunOS, IRIX, Linux, AIX). XSim also added custom graphic model interfaces, on-demand expression graphing, worlds-within-worlds graphics (Harris et al., 1994), remote (client-server) computation and limited multi-processing. JSim development efforts began in 1999 and augmented the functionality developed in SimCon and XSim by adding simplified model specification (using the MML modeling language), facilities for data analysis and for distribution of results and of models (using project files), popular desktop and laptop support (Windows, Macintosh \& Linux) and fully integrated multiprocessing for shared memory systems (Raymond et al., 2003).

\section{JSim overview}

JSim is designed centrally for evaluating models against experimental data, for describing biological systems, for designing experiments, and for the teaching of integrative systems approaches to biological, chemical and physical systems. It is built around a "project file" (.proj), that may hold many data sets, several different models and the results of multiple types of analyses testing models against the data and against each other. JSim's handling of ODEs (ordinary differential equations) suits it for traditional compartmental modeling and SBML (Hucka et al., 2003), CellML (Cuellar et al., 2003), and pharmacokinetic (PK) models in general. Solving PDEs (partial differential equations) hugely expands the range of processes that can be modeled in physiology and clinical medicine (Goresky, 1963; Bassingthwaighte, 1974), biophysics, and PKPD modeling (Roberts \& Rowland, 1986). JSim handles spatial diffusion (Barta et al., 2000; Safford \& Bassingthwaighte, 1977) and convection-diffusion problems. From soon after its release in 1999, JSim provided automated unit consistency checking in all equations and also automated unit conversion (such as minutes to seconds) in calculations (Chizeck et al., 2009). This pair of features automates the first stage of verification of the model's mathematical implementation by making sure that every equation has unitary balance. Modeling taking account of the anatomical quantitative constraints is now recognized as critical and is facilitated by the automated unit checking (Vinnakota \& Bassingthwaighte, 2004). The second phase of compilation parses the details of the equations and sequences them for efficient computation. For an example, a cardiovascularrespiratory system model (Neal \& Bassingthwaighte, 2007), ran under JSim exactly 300 times faster than a Matlab-Simulink version of the identical model (Howard Chizeck/Stephen Hawley: personal communication). In general, using Matlab without Simulink takes 6 to 20 times as long as JSim solutions.

MML (Mathematical Modeling Language) is a declarative modeling language developed for JSim and used for composing models. Its archival version is XMML, in the XML style of SBML and CellML. In MML, one writes mathematical equations directly into the code, and the MML compiler handles converting the set of equations into a sequence of computations. Since the equation representation is closely related to the conceptual formulation of the model, MML models are easily understood, and pieces of the model are readily interpretable as particular processes. The fact that one can write several models into a single MML program allows one to compare competing hypotheses (models). Having a standard layout for graphs and for ASCII text output of model solutions is convenient. For special purposes, as for a model to be used in clinical practice or teaching, an alternative graphical user interface specifically designed for the model can be readily substituted for the default layout.

Declarative languages such as MML describe the logic of a computer program rather than the explicit flow of control. In traditional procedural languages such as $\mathrm{C}$ and Fortran the flow of control is explicit in the code. Declarative languages have advantages and disadvantages relative to procedural languages. They allow for clear exposition of the intentions of computation, since only the equations (without the numerical details) are specified. Because they generally represent a top-down view of the mathematics, they allow automated handling of computational complexities such as parallel processing without distracting the user with complex details. On the other hand, it is difficult for a procedural translation of declarative code, as going from MML to compute using Java, to be as general and efficient as optimized procedural code. Consequently MML is 
designed to permit use of procedural code when circumstances demand it.

\section{JSim problem domain}

JSim is a general purpose simulation and data analysis software system. It handles a wide range of mathematical problems including algebraic equations, ordinary differential equations, and parabolic, hyperbolic and elliptic partial differential equations. It contains 8 ODE and 3 PDE solvers implementing a variety of algorithms which allow the flexibility to strike a balance between accuracy and computational speed. It performs time series analyses including forward and backwards Fourier transforms. MML can handle multi-dimensional PDEs but the solvers currently implemented support only two dimensions (typically time and one spatial dimension). For two spatial dimensions the problem needs to be formulated into either ODE nodes or PDEs in one spatial dimension linked by ODEs in the other spatial dimension. JSim does not support complex numbers or matrix notation and associated matrix operators; in JSim all matrices must be written explicitly as a set of equations.

JSim can be used in any discipline where mathematical equations are used for modeling and analyzing data. JSim was originally developed to model and analyze physiological phenomena and many of the built-in tools were developed to handle physiological problems. But all of the JSim tools can be applied to any other scientific discipline. JSim excels at analyzing time course and spatial domain data in complex systems (Beard \& Bassingthwaighte, 2000; Beard et al., 2005; Bassingthwaighte et al., 2006b; Suenson et al., 1974; Safford \& Bassingthwaighte, 1977). Examples include modeling pharmacokinetic/dynamic (PK/PD), radiological (CT, PET, MRI) and multiple indicator dilution (MID) data.

\section{JSim's mathematical modeling language, MML}

JSim uses the Mathematical Modeling Language (MML) to describe models. When JSim imports other model formats (e.g. SBML, CellML, Antimony (Smith et al., 2013)), it translates them to MML. MML is a concise, ASCII text language for defining parameters and variables and for writing the equations describing a model. MML is a declarative language (as opposed to procedural or imperative languages such as MATLAB, Java, Python, and FORTRAN), meaning that, in MML, equations represent mathematical equality, rather than providing a directive to calculate the left-hand side variable from the expression on the right. In MML, it makes no difference if terms in an equation appear on the left or right hand side. Such equations are a direct representation of the mathematical ideas in a model rather than a procedural formulation. This improves readability and allows for more extensive consistency checks than procedural formulations. The MML compiler checks to ensure that all variables are completely, but not overly, specified - a check unavailable in procedural languages. The compiler sequences the calculations based on the dependencies of the variables to be computed, thus eliminating order-of-operations errors that are possible in procedural languages. MML variables are (optionally) labeled with physical units, enabling the compiler to reject equations with unitary imbalances; this also allows the automated insertion of appropriate unit conversion factors when needed (Chizeck et al., 2009) (e.g. $\mathrm{mmHg}$ to $\mathrm{kPa}$ ). This relieves the modeler of the burden of adding unit conversion factors (another potential source of error) and aids readability, since equations need not be cluttered with conversion factors. MML's design supports the model development and unit balance aspects of modeling steps 2 and 3 above. An example of MML code is shown below as Box 1 , which codes a "progress curve", the concentration-time curves for hypoxanthine to xanthine to uric acid catalyzed by the enzyme xanthine oxidase through the two oxidation steps. MML code for partial differential equations is given in Box 2.

\section{Numeric solvers}

MML is designed without reference to the numerical algorithms that will be used for simulation. Rather, the user selects the numerical methods in the JSim run time user interface. At present JSim provides 8 algorithms for solving ODEs [Table 1] and 3 for PDEs [Table 2]. Numerical methods for stochastic simulation are variants on the Gillespie algorithm (Gillespie, 1977). JSim's solvers support modeling steps 4 to 6 above.

To solve differential equations one needs initial conditions, and JSim's parser (precompiler) demands these, as in Box 1. Partial differential equations also require boundary conditions, as seen in the code for a two-region convection-diffusion-permeation-reaction model [Box 2].

\section{Table 1. JSim ODE solvers.}

\begin{tabular}{|ll}
\hline Auto & $\begin{array}{l}\text { Starts with Dopri5, if Dopri5 fails, switches to Radau } \\
\text { Dopri5 }\end{array}$ \\
$\begin{array}{l}\text { Dormand-Prince explicit Runge-Kutta method of } \\
\text { order 5(4) for non-stiff equations (Hairer et al., 1993) }\end{array}$ \\
\hline Radau & $\begin{array}{l}\text { Implicit Runge-Kutta method (Radau IIA) of } \\
\text { variable order (switches automatically between } \\
\text { orders 5, 9, and 13) (Hairer \& Wanner, 1996) }\end{array}$ \\
\hline KM & $\begin{array}{l}\text { Five stage, 4th order accurate Merson-modified } \\
\text { Runge-Kutta method with adaptive steps (Merson, 1957) }\end{array}$ \\
Fehlberg & $\begin{array}{l}\text { Fifth order accurate Runge-Kutta-Fehlberg Method } \\
\text { with adaptive stepsize, also known as RK45 } \\
\text { (Fehlberg, 1969) }\end{array}$ \\
\hline Euler & $\begin{array}{l}\text { Explicit forward Euler Method, first order accurate } \\
\text { (Euler, 1768; LeVeque, 2007) }\end{array}$ \\
RK2 & $\begin{array}{l}\text { Two-stage explicit Runge-Kutta method, 2nd order } \\
\text { accurate (LeVeque, 2007) }\end{array}$ \\
RK4 & $\begin{array}{l}\text { Classical Runge-Kutta explicit 4th order four-stage } \\
\text { method (LeVeque, 2007) }\end{array}$ \\
\hline CVode & $\begin{array}{l}\text { CVODE, a publicly available stiff ODE solver } \\
\text { (Cohen \& Hindmarsh, 1995) }\end{array}$ \\
\hline
\end{tabular}

Table 2. JSim PDE solvers.

\begin{tabular}{ll} 
& $\begin{array}{l}\text { Lagrangian Sliding Fluid Element Algorithm } \\
\text { (Bassingthwaighte, 1974; Bassingthwaighte } \\
\text { et al., 1992; Poulain et al., 1997). The convecting } \\
\text { step is solved separately from the other } \\
\text { processes }\end{array}$ \\
LSFEA & $\begin{array}{l}\text { 2nd order accurate finite difference method } \\
\text { for solving hyperbolic differential equations } \\
\text { (MacCormack, 1969) }\end{array}$ \\
MacCormack & $\begin{array}{l}\text { Finite element discretization akin to a nonlinear } \\
\text { Galerkin method 2nd order accurate in space } \\
\text { (Blom \& Zegeling, 1994) }\end{array}$ \\
\hline
\end{tabular}




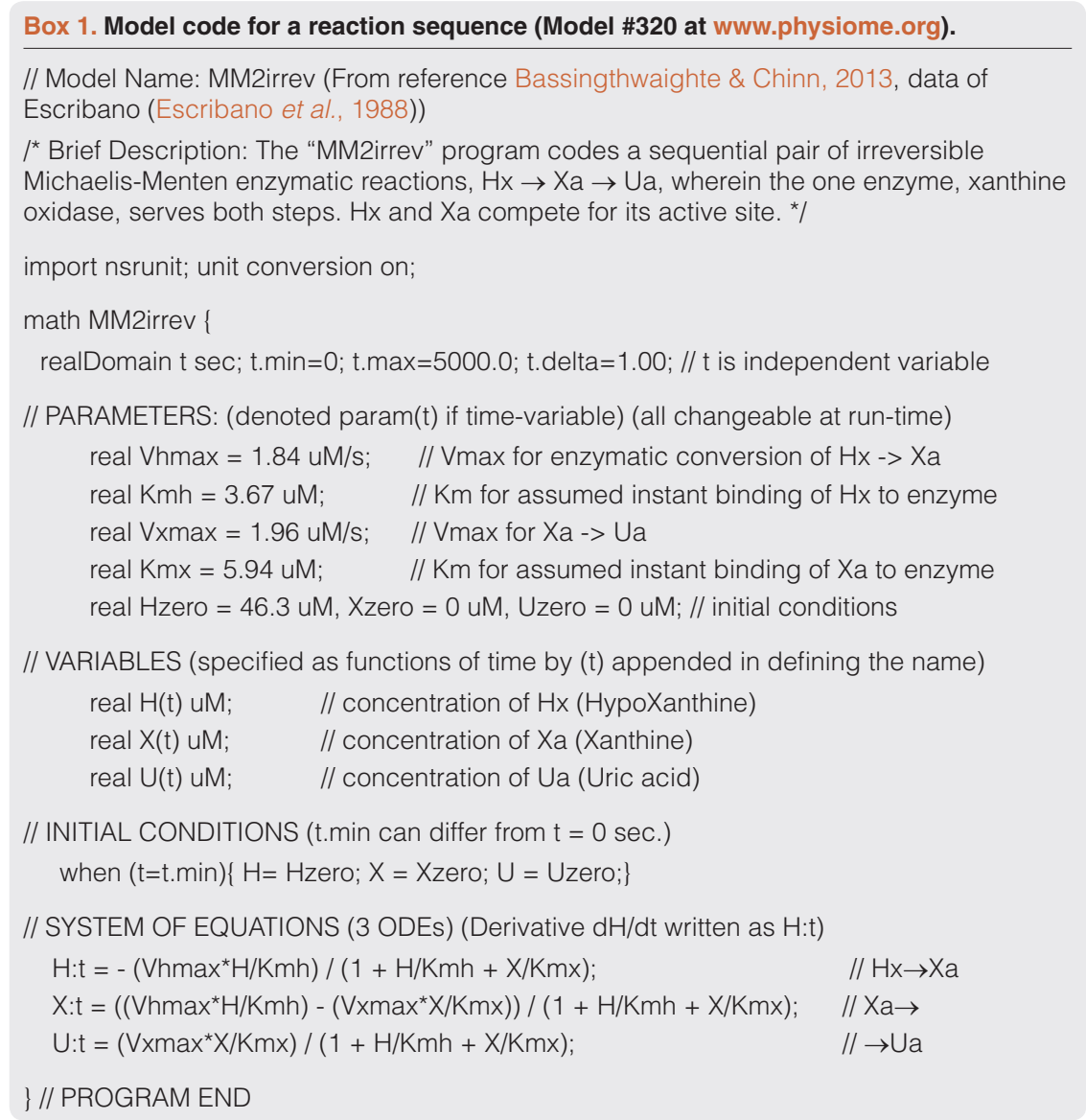

\section{Function generators}

Many physiological systems or components (e.g. one for the uptake of a metabolite) can be considered as operators. The operator takes an input function (e.g. inflowing solute concentration) and produces an output function (e.g. outflowing solute and metabolite concentrations). Model behavior can be tested by using various input waveforms (e.g. as in Box 2 "extern real Cin(t)") described by JSim "function generators". These might be time series signals of diverse form (pulses, pulse combinations, sines, shaped sawtooth), probability density functions (Gaussian, exponential, Poisson, lognormal, gamma variate, random walk, etc.), or come directly from experimental data. When there is no consumption and the system is linear (output area equals input) and stationary (response same at another time), then the output function is the convolution of the operator's transfer function (the response to an infinitely short pulse input) with the input function. Users select input functions at run time for testing numerical algorithms for correctness (verification testing), for model exploration (behavioral analysis) or for analyzing data as for steps 6 and 7 in our " 13 -Step" process.

\section{Model behavioral analysis and visualization}

We will use a simple convection-diffusion reaction model (Bassingthwaighte, 1974; Bassingthwaighte \& Goresky, 1984) to illustrate some facilities for visualizing model solutions and the effect of varying parameter values on them. The system is diagrammed in Figure 1 and the code is provided in Box 2.

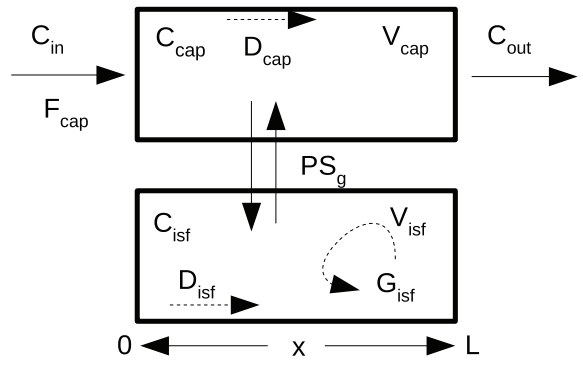

Figure 1. Capillary-tissue exchange unit. Fluid flows with velocity $F_{\text {cap }}{ }^{*} L / N_{\text {cap }}$ along the capillary from the entrance at $x=0$ to the exit at $\mathrm{X}=\mathrm{L}$, and exchanges across the capillary wall into a stagnant extravascular region with conductance PS, the permeability-surface area product. The input is a bolus of solute, $\mathrm{C}_{\text {in }}(\mathrm{t})$, entering the capillary with the flow, $F_{\text {cao }}$. Axial gradients along the capillary are diminished by diffusion, $D_{\text {p }}$ and $D_{\text {ist }}$. Tissue consumption occurs at rate $G_{\text {isf }}{ }^{*} C_{\text {isf }}$. This is a simplified version of models used for indicator dilution studies and PET clinical studies (Beard \& Bassingthwaighte, 2000; Bassingthwaighte et al., 1989; Bassingthwaighte et al., 1992; Bassingthwaighte et al., 2006b). 


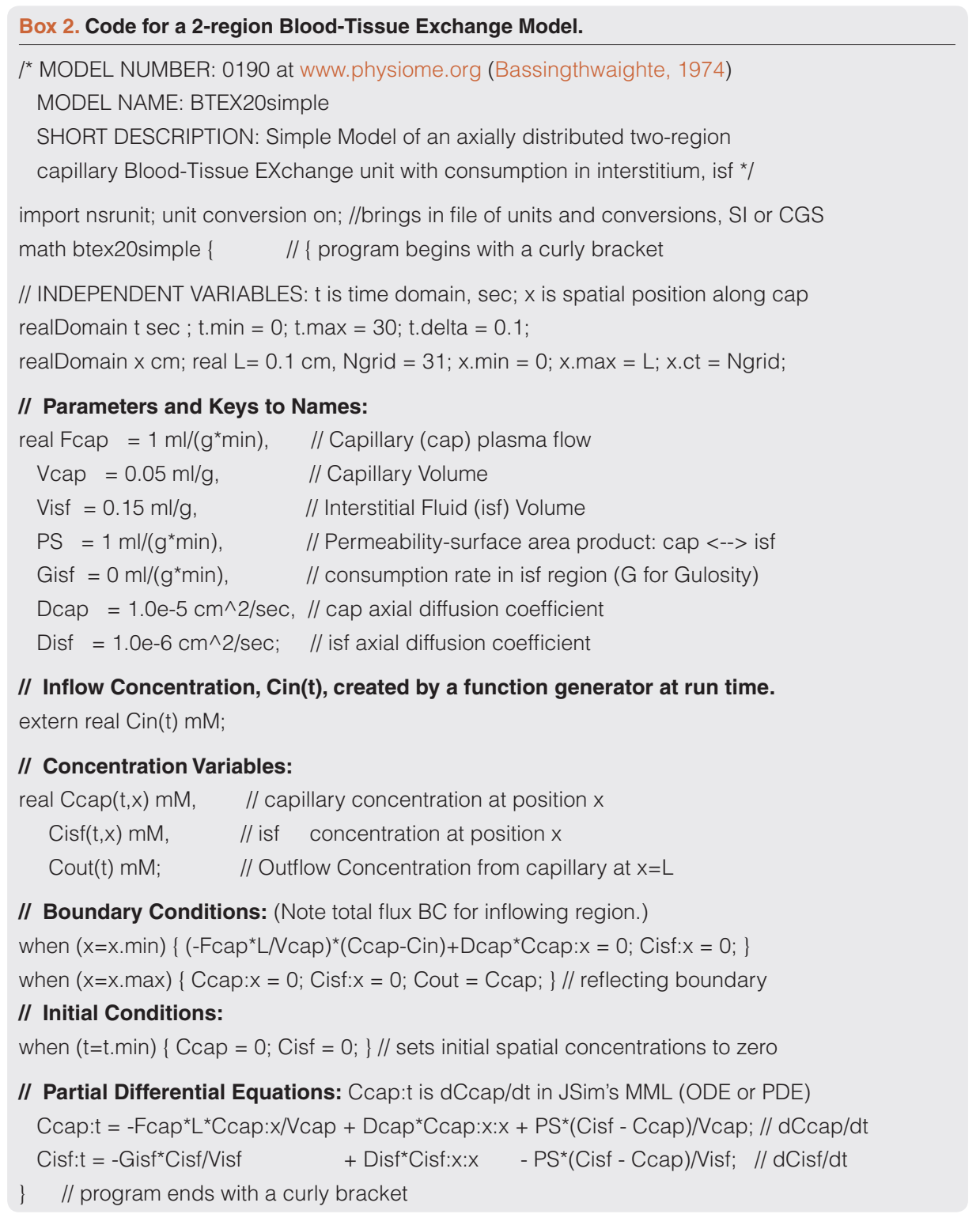

\section{Plot pages}

JSim provides several mechanisms for visualization, providing insight about model dynamics. The most basic are plot pages, each of which may contain line, scatter, contour and colormap plots. One may plot experimental data and model solutions (from one or more models), scaled automatically or manually, linear or logarithmic, plotted as they are being computed or displayed or edited later. Multiple plot page configurations are stored in each project, enabling reproducible analysis (e.g. all the data and graphs for a particular journal article). JSim plot pages support modeling steps 1, $5,6,7,8$ and 10 above (display of experimental data and model solutions, verify solution accuracy, explore model behavior, display of sensitivity curves and assessments of goodness of fit).
LOOPS: Iterating solutions to exhibit behavior

Model loops are a feature for behavioral analysis that plot data from a family of model runs using a user-chosen sequence of parameter values. For example, Figure 2, "looping over", i.e. making a sequence of changes in a parameter value for the membrane permeability in a tracer uptake model yields a family of plots showing how outflow tracer concentration curves would vary with varying permeability. The curves, of course, depend upon the settings for the other parameters of the model, so the looping sequence should be initiated under widely divergent conditions in order to understand the "conditions" (the regions of state space) where the chosen parameter may have little influence or maximum influence. JSim's loops facility support modeling steps 6 and 7 above (verify solution 


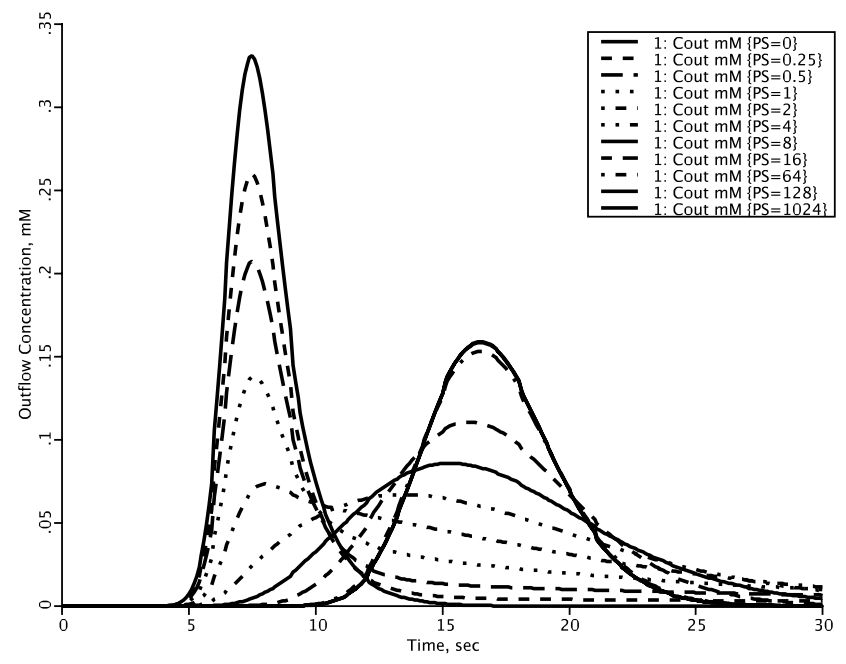

Figure 2. Using LOOPS to explore parameter influences. [Model is in Figure 1, with the computer code in Box 2.] With the permeability surface area product (PS) $=0$ (tallest solid curve) in the axially distributed capillary-tissue model in Figure 1, the outflow concentration-time curve, $\mathrm{C}_{\text {out }}$, represents the response function through the vascular space alone. The mean transit time for this curve is $\mathrm{V}_{\text {cap }} / \mathrm{F}_{\text {cap }}$. With finite permeability, PS, there is extraction of solute during transcapillary passage, shown by the successive diminutions of the heights of the initial peaks as PS increases. At low PSs the form of the outflow begins for a second or so as a reduced version of the curve with $P S=0$. At low PSs the flux into the tissue is purely "barrier limited", i.e. the permeation of the barrier has the dominant influence on the shape of the outflow curve. When PS is 4 or greater $\mathrm{ml} /\left(\mathrm{g}^{*} \mathrm{~min}\right)$, the sixth curve, the initial peak is no longer discernable; at yet higher PSs a second peak arises, and at PSs above $128 \mathrm{ml} /\left(\mathrm{g}^{*} \mathrm{~min}\right)$ increasing the PS further has no effect on the shape of the outflow curve and at high PSs the exchange flux is purely "flow-limited", where changing the flow shifts $\mathrm{C}_{\text {out }}$, but changing PS does not. When PS $>0$, then the mean transit time for all the curves is $\left(V_{\text {cap }}+V_{\text {isf }}\right) / F_{\text {cap }}$., Thus there is a gradual shift from being purely barrier-limited, through a mixed barrier- and flowlimited regime to a purely flow-limited regime at high PS where the shape of the curve is influenced only by changes in flow, but the mean transit time is unchanged over the whole range, PS $>0$ to $\mathrm{PS}=1024 \mathrm{ml} /\left(\mathrm{g}^{*} \mathrm{~min}\right)$, being obedient to the laws of conservation of mass.

accuracy, explore model state space). A convenient feature of the LOOPS function is that the user can stop the solution, automatically starting the next one, whenever desired, speeding up the review of solutions. This is especially important in large models with long computation times. "Inner" and "outer" loops provide two levels of nesting, when needed.

\section{Nested plots}

Nested plots [Figure 3] are JSim's version of worlds-within-worlds graphics (Harris et al., 1994). Each nested plot is a 2-dimensional array of plots, each of which represents the form of a set of model solutions with a pair of distinct parameter value. Nested plots enable simultaneous visualization of the effect of up to six independently varying parameters. JSim nested plots support modeling steps $6-8$ above (verify accuracy, explore model state space, sensitivity analysis).

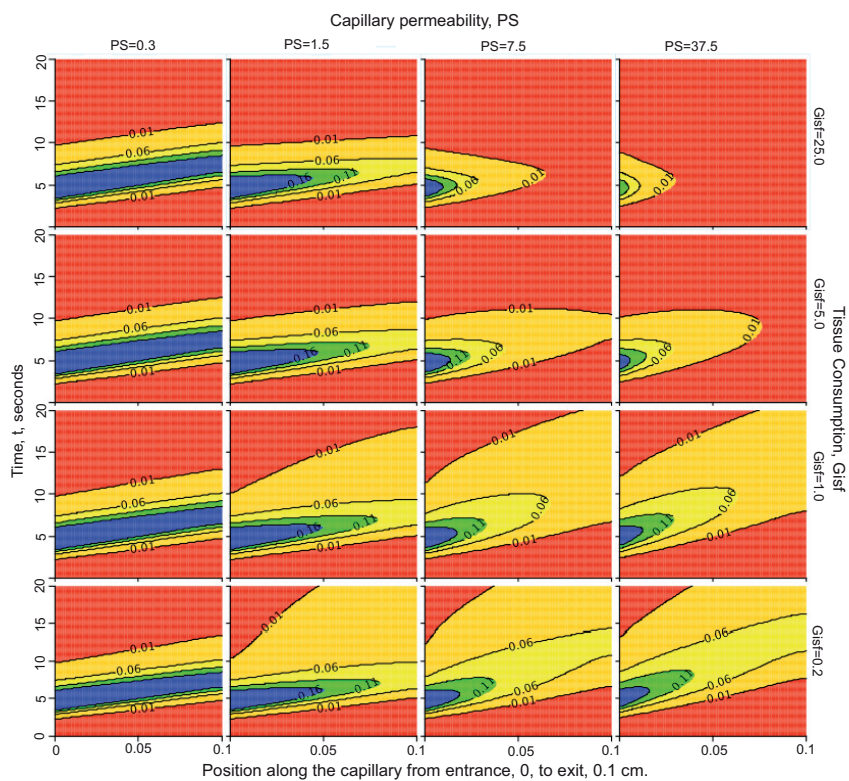

Figure 3. Nested plots. Behavior of the two-region model when varying capillary permeability, $P S$, and tissue consumption, $G_{\text {ist. }}$ Each panel is a contour plot: the abscissa is position $x$ between the capillary entrance at $x=0$ to the exit at $x=0.1 \mathrm{~cm}$; the ordinate is time, $t$. At each time t a horizontal line from 0 to 20 is colored (using color profile "rainbow" in this case) in accord with the concentration (highest is blue, to which each contour plot is scaled, down to red for zero concentration) at each point in $x$. Convection moves the entering solute along the tube from left to right to larger $x$ on this graph. With successive times the colored horizontal lines construct a shaped profile above the $x$ - $t$ plane; contour lines with units in $\mathrm{mM}$ are superimposed. The columns from left to right show contours with $P S$ increasing by factors of 5 (see labels at top of column) from $P S=0.3$ to $37.5 \mathrm{ml} /\left(\mathrm{g}^{*} \mathrm{~min}\right)$. The rows go from consumption $G_{\text {isf }}=0.2 \mathrm{ml} /\left(g^{*} \mathrm{~min}\right)$ in the bottom row by factors of 5 to the top row with $G_{\text {isf }}=25 \mathrm{ml} /\left(g^{*} \min \right)$; see labels on right ordinate. With low $P S$, leftmost column, as with the low PS outflow curves at Ccap $(t, x=L)$ or Cout(t) of Figure 2, very little of the solute escapes into the tissue, so the injected bolus remains relatively compact even while undergoing some diffusional spread $\left(D_{\text {cap }}=D_{\text {iff }}=10^{-4} \mathrm{~cm}^{2} / \mathrm{sec}\right)$, and the influence of the consumption is negligible since so little enters the ISF. With increasing PS more solute enters the ISF where it is consumed. With high $P S$ and high $G_{\text {isf }}$, the right uppermost panel, the solute is all consumed before it can reach the capillary exit at the right edge of the panel. [This plot is set up under "Project", "Add", "New Nested Plot" using LOOPS, inner and outer, to set the values for the parameters, and on the NestedPlot, then clicking on "XY plot" to choose "contour". Instructions are under Running JSim - Data Analysis - Nested plots: www/physiome.org/jsim/docs/User.html].

\section{Sensitivity analysis}

By "sensitivity analysis" we mean the examination of the influences of individual parameters on the model responses under a wide variety of conditions. The sensitivity function, $S(t)$ is the change in magnitude, $\mathrm{dQ}$, of variable $\mathrm{Q}$, to a small change in a parameter value, $d P$. It may be expressed in a normalized form, $(\mathrm{dQ} / \mathrm{Q}) /(\mathrm{dP} / \mathrm{P})$, or unnormalized form, $\mathrm{dQ} / \mathrm{dP}$. As an example consider the same model as was explored in Figure 3. Figure 4 shows the sensitivities of the outflow concentration of a solute to a change in interstitial 

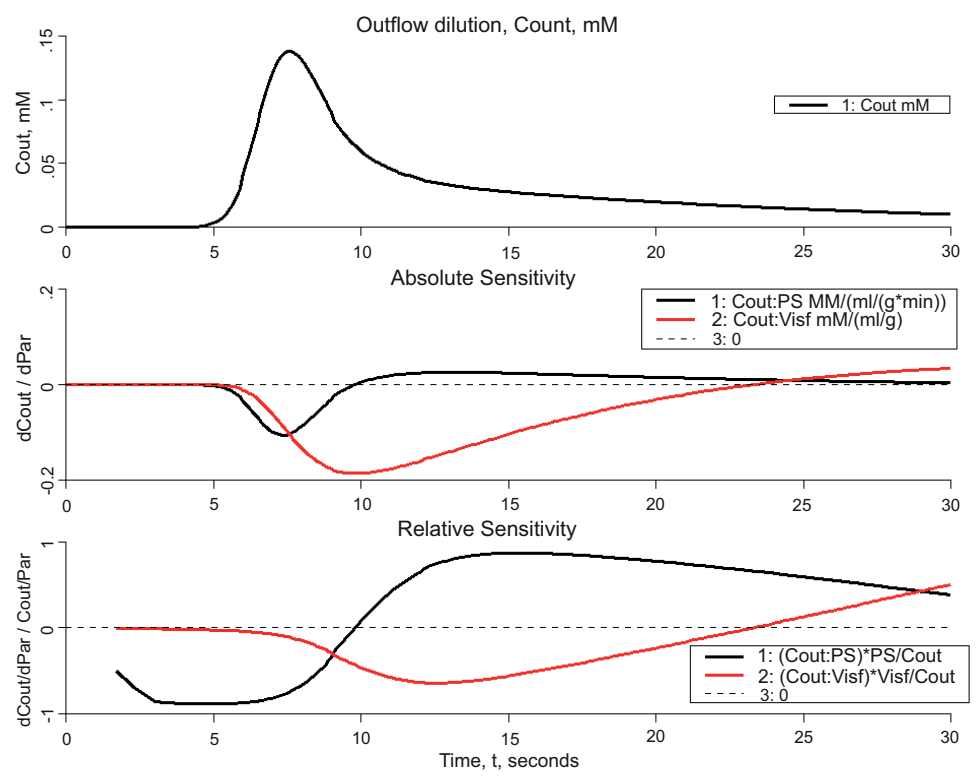

Figure 4. Sensitivity analysis using the same model as in Box 2 and Figure 2 and Figure 3. Upper panel: Model solution for outflow from capillary. Parameters were as in Box 2, the default parameters. Middle panel: Sensitivity function, $d f / d p$, the change in $\mathrm{C}_{\text {out }}$ with a $1 \%$ increase in the capillary wall conductance (PS), black curve or the interstitial volume $\left(\mathrm{V}_{\text {isf }}\right)$. Lower panel: Normalized sensitivity function, $(d f / f) /(d p / p)$, the fractional change in $\mathrm{C}_{\text {out }}$ divided by the fractional change in each parameter, again for a $1 \%$ change in the parameter value.

fluid volume $\left(\mathrm{V}_{\mathrm{isf}}\right)$ or capillary wall conductance $(\mathrm{PS})$ following an injection of that solute at the capillary entrance. The upper panel shows the outflow concentration without parameter perturbation. The middle panel plots the unnormalized sensitivity functions, and the bottom plot shows the normalized sensitivity functions (with the early part of the curves removed when $\mathrm{C}_{\text {out }}$ is negligible). Increasing PS will lower the height of $\mathrm{C}_{\text {out }}$ for the first 10 seconds with the greatest reduction at the peak of $\mathrm{C}_{\text {out }}$ at $\sim 8$ seconds (due to greater flux of metabolite into the ISF); after 10 seconds, the height of $\mathrm{C}_{\text {out }}$ will be increased (back flux of metabolite from ISF). Increasing $V_{\text {isf }}$ has the effect of lowering $\mathrm{C}_{\text {out }}$ for the first 24 seconds, then raising it after 24 seconds. JSim's sensitivity analysis supports modeling step 7 above.

\section{Optimization}

Manual parameter adjustment to fit the model to experimental data is encouraged as a means of gaining insight into model behavior. Automated parameter optimization is usually much faster; eight methods are provided [See Table 3]; we recommend testing several in order to test speed and reliability with respect to the particular

\section{Table 3. JSim's optimizers.}

$\begin{array}{ll}\text { Simplex } & \begin{array}{l}\text { A bounded, non-linear steepest-descent algorithm (Dantzig et al., 1955) } \\ \text { Derivative-free non-linear optimizer. Uses adjustable mesh and linear least } \\ \text { squares to find smoothed values of function, gradient and Hessian at center } \\ \text { of mesh. Values drive a descent method that estimates optimal parameters, } \\ \text { but is unbounded (Bassingthwaighte et al., 1988) }\end{array} \\ \text { GGopt } & \begin{array}{l}\text { A bounded, parallel algorithm. Operates via progressively restricted search } \\ \text { of parameter space on a regularly spaced grid of N points per dimension } \\ \text { (Kolda et al., 2003) }\end{array} \\ \text { GridSearch } & \begin{array}{l}\text { Unbounded, steepest descent similar to Simplex (Nelder \& Mead, 1965) } \\ \text { NelderMead }\end{array} \\ \text { NL2SOL } & \begin{array}{l}\text { An adaptive nonlinear least-squares algorithm (Dennis et al., 1981; Dennis \& } \\ \text { Schnabel, 1983) Unbounded }\end{array} \\ \text { SENSOP } & \begin{array}{l}\text { A weighted nonlinear least squares optimizer using a variant of the } \\ \text { Levenberg-Marquardt method to calculate the direction and the length of the } \\ \text { step change in the parameter vector (Chan et al., 1993) Bounded }\end{array} \\ \text { SimAnneal } & \begin{array}{l}\text { Simulated annealing for finding the global optimum of a function in a large } \\ \text { multi-dimensional parameter search space which is first randomly sampled } \\ \text { with step-size decreasing with time (Kirkpatrick et al., 1983) }\end{array} \\ \text { Genetic } & \begin{array}{l}\text { Genetic algorithms are a family of algorithms that generate a population of } \\ \text { candidate solutions selecting the best solutions in each iteration to "mutate" } \\ \text { and "cross over", creating a new generation of solutions in an iterative } \\ \text { process (Holland, 1992) }\end{array}\end{array}$


types of data and model. Given that some parameters are known or highly constrained, one may obtain the best model fit to the data for a particular subset of model parameters, and one may also, for some but not all of the optimizers, constrain the range for each parameter value, applying scientific judgment. Optimization helps in finding systematic misfits to the data (and the possible rejection of the hypothesis), and in estimating parameter values.

The optimizer works to minimize an objective function, usually a weighted sum of squares of the differences between the model solution and the experimental data at each observation time or spatial position. This may require freeing up most parameters for optimization to make sure that an assumed constraint isn't creating a biased solution. JSim provides a graph of residuals (the differences between model and data); sign tests and other statistical appraisals of the residuals as a function of time help to distinguish systematic from random deviations. JSim's optimization facilities support modeling steps 9-12 above (fitting solutions, assessing goodness of fit, examining parameter correlations, evaluating confidence limits).

\section{Parameter confidence ranges}

Model fitting to the data is never unique but is guided by the weighting of the observed data points and the noise in the data. Parameter estimates are not exact, but merely estimated, and even possibly biased by the user's choice of the weights on individual data points. How to obtain a "best fit" of model function to data is always, in a sense, a personal choice. Guidelines include weighting inversely to the likely standard deviation of each data point, or unweighting outliers. Viewing the graph of residuals (the differences between data and model) is most helpful in identifying systematic misfits.

Ignoring how one got to the point of "best fit", one desires an evaluation of the parameter values. If the optimized parameters do generate outputs that closely match the experimental data, the question becomes what confidence can be placed on these estimates. One simple method is to optimize using several different numerical method, i.e. different optimization algorithms and different weighting schemes, to see how much the "best fit" parameter estimates change. Other methods of estimating parameter confidence limits include using the Jacobian and using Monte Carlo methods.
Using the Jacobian: The Jacobian matrix is the matrix of the sensitivity functions for all the parameters open to optimization, as calculated at the location of the minimized objective function, the "best fit". This matrix, which JSim calculates after each optimization provides the basis for determining correlations among parameters, and the confidence limits (standard deviations and expected ranges based on Gaussian assumptions). The calculation assumes symmetry and linearity, and so makes only local calculations, and gives no guarantee that the "best fit" is a global best fit. While getting to the "best fit" point in parameter space is data-dependent, this confidence range estimation procedure is not at all, for it is estimated solely from the shapes of the local sensitivity functions. Thus it behooves one to get the differing estimates obtained from different optimizers, different numbers of parameters searched, and even to move the parameter "best fit" values a little away from the optimizer's choice and recalculate the confidence ranges.

Using a Monte Carlo method: A more robust, but more demanding, confidence limit calculation uses Monte Carlo methods. The procedure is to 1) Select a noise profile for each experimental data point, ideally based on what you believe the real noise is, e.g. 5\% proportional Gaussian random noise. 2) Generate a perturbation for each experimental data point by drawing randomly from the selected noise profile. 3) "Run" Monte Carlo automatically re-optimizes the model against the new set of perturbed data points to obtain another estimate for each parameter, repeating steps 2 and 3 many times (e.g. set it to 1000). From these many results, one obtains a histogram of estimates for each optimized parameter, and robust confidence limits can be drawn directly from these histograms without assuming symmetry and linearity as in the Jacobian method. JSim also displays these results in the form of 2-parameter scatter plots to show covariance. Given that the model code has been verified for mathematical accuracy over the full range of parameter space used for the Monte Carlo evaluation, the result gives an informative measure of the degree of validity of the model. This is the first step of uncertainty quantification (Smith, 2014).

\section{Network graphs}

JSim's model "browser" provides a visual representation of model variables as "nodes" and their dependencies or connectivity with each other as connecting lines or "edges". See Figure 5. The graphs

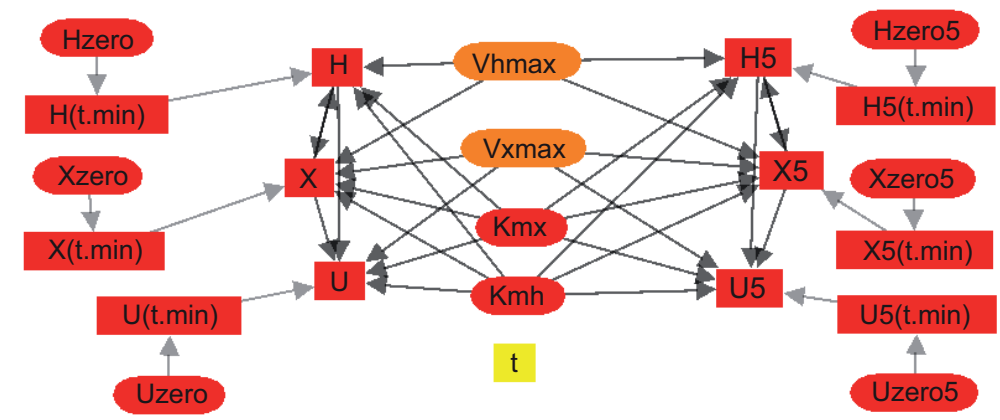

Figure 5. Connectivity graph for a modified version of the model program in Box 1. For $\mathrm{Hx} \rightarrow \mathrm{Xa} \rightarrow \mathrm{Ua}$, the oxidation of hypoxanthine to xanthine to uric acid, catalyzed by xanthine oxidase. The connectivity is shown for a dual solution version of the code for fitting two different sets of experimental data simultaneously with a common group of parameters so as to obtain a minimally biased set of parameter confidence ranges. 
can be selected to include model parameters, or selected classes of variables, e.g. pressures, strains, concentrations. This feature is based on work by Yngve (Yngve et al., 2007). JSim's model browser supports modeling step 2 above (development of the model).

\section{Implementation}

JSim is implemented in the Java computer language (Gosling \& McGilton, 1996). The major factors affecting this choice are Java's platform independent GUI (allowing Windows, Macintosh and Linux versions to be developed in a single code base), object-oriented features and garbage collection (simplifying complex coding), advanced utilities (associative arrays, dynamic linking, remote procedure calls), strong type checking (allowing many common coding errors to be caught at compile time) and robust exception mechanism (simplifying coding and enabling a virtually crash-proof GUI). Native code (C and Fortran) is used in certain restricted circumstances using the Java Native Interface (JNI) (Liang, 1999) to reduce computational overhead (transcendental functions, 2D array access) and the availability of legacy code libraries (ODE, PDE and optimization numerical methods).

The MML language is parsed using JLex scanner generator and the CUP parser generator (Appel, 1998). These tools, similar to the classic Unix lex and yacc (Aho \& Sethi, 1988), were among the few parser generation tools available for Java when JSim was first developed. Using a formal parser generator allows MML to be concise, intuitive, consistent and extensible. MML's declarative structure is an intuitive expression of a model's underlying mathematics (simplifying the modeler's learning) and allows the overall structure of the model to be examined for mathematical correctness (detecting overspecification or underspecification) in a way that is not possible with a procedural specification. Units and unit checking (Chizeck et al., 2009) were added to MML soon after its initial design to further improve model conciseness and assure unit balance in the equations as a first step in verifying that the mathematics is rendered correctly by the numerics.

MML is compiled into Java model computational code for run-time execution. This results in faster model execution (in comparison to table-driven computations) and allows more flexible model computational structure (multiple time sweeps, indexed loops). JSim models run asynchronously to the GUI in contrast to most simulators which alternate computational and graphical update steps. This approach dramatically improves performance and user response, especially when remote computation is used. JSim's remote computation is implemented using Java Remote Method Invocation (RMI) (Harold, 1997), providing reliable access to networked computational servers. This approach also isolates the JNI methods (above) in the computational engine, allowing the JSim GUI to run as a pure Java browser applet. JSim multiprocessing is implemented using Java threads (Oaks \& Wong, 2004) providing excellent performance and seamless integration with the Java memory management and exception mechanisms (providing application stability). MML code is stored as XMML for distribution, and has automated translators into XMML, SBML, CellML, and with limitations into Matlab (Smith et al., 2013).

\section{Run-time performance}

Run-time performance of JSim is dependent on numerous factors: model complexity, mathematical formulation, numeric methods used, use of parallel processing, and the fineness of time and spatial grids used. PDEs generally run faster than ODEs providing similar spatial resolution even though they include diffusion terms, but in general can be slower than ODEs representing simplified geometries, and are slower using high resolution general purpose solvers like TOMS731. A direct solver-to-solver comparison on a problem which can be formulated as either an ODE or PDE model (a convection-diffusion model) is provided in Table 4.

The times reported in Table 4 are extraordinarily variable, and depend upon parameter values and on the values of the variable themselves, as the solvers can become highly efficient if a variable is not changing. Naturally, variables solved explicitly tend to run faster than those solved implicitly. But there are exceptions to this, for example solutions to PDEs for capillary-tissue exchange that require convolutions and double convolutions over Bessel functions take over a million times as long as the PDE solvers (Bassingthwaighte et al., 1989). In general, linear systems of implicit equations run faster than non-linear systems. Runs requiring stiff ODE solvers (e.g. CVode, Radau) typically run slower than non-stiff solvers (e.g. Dopri5, RK4). Analyses involving JSim loops, sensitivity analysis, optimization and Monte Carlo methods run faster when JSim multiprocessing is activated. Models requiring fine temporal or spatial grids to capture relevant detail run slower than those for which coarse grids are sufficient.

Computation time equivalent to real time was shown on a laptop computer for a cardiorespiratory system model with about 120 variables (Neal \& Bassingthwaighte, 2007). Models available at physiome.org typically run somewhere between a fraction of a second to several minutes, depending upon these various complications. Some example model simulation execution times ("run times") are compared to the real time duration of the events being modeled ("model times") in Table 5. All the runs below were single

Table 4. JSim's Optimizers.

\begin{tabular}{|llll}
\hline Solver name & $\begin{array}{l}\text { Solver } \\
\text { type }\end{array}$ & $\begin{array}{l}\text { Mathematical } \\
\text { formulation }\end{array}$ & $\begin{array}{l}\text { Run time, sec } \\
\text { (single simulation } \\
\text { run, 120 sec of } \\
\text { a convection- } \\
\text { diffusion model } \\
\text { with } \Delta \mathbf{t}=\mathbf{0 . 1} \text { ) }\end{array}$ \\
\hline LSFEA & PDE & PDE & 285 \\
\hline TOMS731 & PDE & PDE & 8155 \\
\hline Radau & Stiff ODE & ODE & 430 \\
\hline CVode & Stiff ODE & ODE & 281 \\
\hline $\begin{array}{l}\text { Dopri5(RK5 } \\
\text { varystep) }\end{array}$ & ODE & ODE & 48 \\
\hline $\begin{array}{l}\text { RK Fehlberg } \\
\text { (RKF45) }\end{array}$ & ODE & ODE & 325 \\
\hline RK Merson & ODE & ODE & 32 \\
\hline
\end{tabular}


Table 5. Solution Times for Some Models.

\begin{tabular}{|llll|}
\hline Model & $\begin{array}{l}\text { Model } \\
\text { time }\end{array}$ & $\begin{array}{l}\text { Run } \\
\text { time }\end{array}$ & $\begin{array}{l}\text { Model time } \\
\text { per. run time }\end{array}$ \\
\hline $\begin{array}{l}\text { Box1 (reaction sequence) } \\
\text { Box2 (two-region blood- } \\
\text { tissue exchange PDE) }\end{array}$ & $3000 \mathrm{sec}$ & $356 \mathrm{~ms}$ & 14000 x faster \\
$\begin{array}{l}\text { Comp2 (two-region blood- } \\
\text { tissue exchange ODE) }\end{array}$ & $30 \mathrm{sec}$ & $130 \mathrm{~ms}$ & $230 \times$ faster \\
\hline $\begin{array}{l}\text { Beeler-Reuter (cardiac } \\
\text { action potential; } \Delta \mathrm{t}=0.1 \mathrm{~ms})\end{array}$ & $600 \mathrm{~ms}$ & $636 \mathrm{~ms}$ & same \\
\hline $\begin{array}{l}\text { Beeler-Reuter (cardiac } \\
\text { action potential; } \Delta \mathrm{t}=0.01)\end{array}$ & $600 \mathrm{~ms}$ & $\begin{array}{l}3059 \\
\mathrm{~ms}\end{array}$ & $5 \times$ slower \\
$\begin{array}{l}\text { Winslow-Rice-Jafri (action } \\
\text { potential; } \Delta \mathrm{t}=1 \mathrm{~ms} \text { ) }\end{array}$ & $1200 \mathrm{~ms}$ & $983 \mathrm{~ms}$ & $19 \%$ faster \\
\hline
\end{tabular}

model runs performed on a mid-range workstation (Dell Precision T3500 Xeon x86-64 2.5GHz). The Beeler-Reuter action potential model (\#78 at physiome.org) has only 4 ionic currents and 28 timedependent variables; the (Winslow et al., 1999) model (\#217) has 126 time-dependent variables. Timing calculations are unreliable, dependent on the model, computational methods, and the values of the variables, and the timestep length. For the Winslow et al., 1999 model, using a 1 microsec timestep took 96 seconds, only 100 times slower, not 1000 times, compared to that with $\Delta \mathrm{t}=1 \mathrm{~ms}$.

\section{Code verification}

We use a variety of strategies to verify the JSim code stack. Some calculations such as values for transcendental functions and algebraic expansion of symbolic derivatives have known closed form solutions that can be compared exactly. Our general policy is to write the code for analytical solutions into the JSim model to use the comparison to verify specifiable cases. In simple cases, e.g. respiratory mechanics models, exponential equations match numerical solutions for 7 decimal points. Some ODE and PDE models such as exponential washout have known closed form analytic solutions which can be numerically compared to solutions generated by JSim's numeric integrators. Even when a complete analytic solution is not available, certain statistics of the solution, such as mean transit time in blood-tissue flow models, can be calculated analytically and compared with the same statistic calculated from the model output.

Parameter changes to models causing output changes that don't correspond to expectation from induction, need to be evaluated qualitatively by the user. When modelers observe unexpected behavior, checking at deeper levels is required. While most such anomalies are due to user coding errors, over the 15 years of JSim's existence, some subtle computation bugs in JSim have been diagnosed in this manner. JSim cannot be proven bug-free even though finding anomalies is now rare: queries regarding problems in computation are welcomed at staff@physiome.org.

JSim models are sometimes exported in SBML format and run in other SBML supporting simulators as a comparison check. Models are also sometimes entirely recoded in a different computational environment (Matlab is often used) as a comparison check. Model translations to other languages (e.g. SBML, CellML, Antimony) are verified via round-tripping (exporting to the target language and then reimporting).

Tests of JSim's optimization and Monte Carlo functionality are based on convergence to solutions of known parameterization. The optimizers are all very different; we advocate that users try a variety of optimizers for any given problem. There is no magic in an optimizer; the key in fitting a model solution to data, when the data are reliable, is in designing a carefully weighted distance function to fit as many computed model variables to simultaneously obtained experimental data functions as possible.

Tests of JSim's multi-processing are based on comparisons to single processor computations.

The JSim verification suite consists of over 1200 individual tests drawn from the above methodologies. The suite is expanded when new computational or translation facilities are added, or when a bug has been found and fixed. Most tests consist of comparing jsbatch output with user-verified reference data. The verification suite is run before every official JSim release to ensure consistency of operation.

\section{Reproducibility}

The all-too-frequent failures to reproduce published results are a critical problem in advancing the biological sciences. It is easy to understand that biological laboratory studies, with inherently great variability in materials and analysis procedures, should be less exact than those in the physical sciences, but it is not so forgivable that reproducing mathematical models of biological systems is a major problem. The two major repositories of published biological models, Biomodels (http://www.ebi.ac.uk/biomodels-main/) using SBML (www.sbml.org) and CellML (models.cellml.org), together have about 1000 curated models: there were errors in the publications requiring corrections in all but 5 of these, before the models could be demonstrated to run appropriately. These models all used algebraic, ODEs, or differential-algebraic equations and so must be regarded as relatively simple, computationally, compared to finite-element models or spatially dependent models. That only $0.5 \%$ of the not very complex models were reproducible is truly alarming, and demonstrates the lack of dedication to making scientific advances useful to others. Some open access journals, such as F1000Research, are aiming to improve this sad state, by requiring open source code to be deposited, hopefully along with the data that provide tests of the model hypotheses. A Special Section in Science (Stone \& Jasny, 2013) is devoted to the issues of open access, addressing open access, peer review, the changing publishing scenario, and encouraging broader methods of communication.

\section{Project files as vehicles for reproducible modeling and data} analysis

JSim project files store a set of codes for models, illustrative figures or diagrams, parameter sets, multiple data sets, the settings for looping, sensitivities, behavioral analysis, and optimizations, plot page configurations, and for project notes. Many models in the Physiome Repository (most of which are JSim-based) have experimental data in the project files for validation testing. Project 
files support the reproduction of a set of simulations and analyses for their sharing across JSim's supported platforms (Windows, MacOS, Linux). Project files support the modeling steps 1 and 13 above (from importation of data, to preservation and distribution of analyses). The MML, XMML and all the data and analyses are preserved in an ASCII format; thus the files tend to be small. The models described above take $<100 \mathrm{kB}$; large models with several hundred ODEs take up $<500 \mathrm{kB}$ even with large time series of physiological data. These files are all human readable, and ready to run when opened in JSim. They contain everything used by the program: the notes, the source code, and the control parameters for all the steps in the analysis. They are editable in any word processor, but one avoids doing that since it is easier to enter code and notes under JSim editor directly and not disturb the format in the XMML file that JSim reads.

There are many models on the Physiome Repository (www.physiome.org) with multiple data sets, model fits to data, and optimization results. Examples are that of Kuikka et al on glucose uptake by myocardium (Kuikka et al., 1986, models 163 and 173), xanthine oxidase reactions (Bassingthwaighte \& Chinn, 2013, model 324), and lung endothelial serotonin uptake (Jardine \& Bassingthwaighte, 2013, model 198). All the JSim project files are stored in a Concurrent Versions System (CVS) archive so that the latest versions, as well as older versions, are always available. The models themselves are copyrighted but researchers are given the freedom to download, modify, and to construct new models from them so long as original authorship is acknowledged.

\section{Modeling over the web}

The approximately $400 \mathrm{JSim}$ models archived at www.physiome. org can be run over the web, with complete freedom to vary the parameters, modify the code, compile and run, reanalyze data that are there in the project file, view residuals, confidence ranges, use Monte Carlo to reveal parameter variances and correlations, etc. (Models based on MATLAB or FORTRAN, a small fraction of the repository, cannot be run over the web but can be downloaded).

\section{Using archived models for analyzing one's own data}

All the archived models may be downloaded so that an experimentalist can import his own data into the project file and analyze it. The JSim Home Page is an operations manual for downloading, running models and analyzing data. For parameter evaluation the number of trial optimizations can be set to 1 (so there is no optimization done) but the covariance matrix is calculated to provide estimates of confidence limits from the local linear combination of sensitivity functions. For greater generality one should set up the optimizer to evaluate the set of parameters desired, then run the Monte Carlo (tab at bottom) to repeat the optimization many times in the presence of added noise; this provides realistic probability density functions of parameter values.

\section{Some Alternative Simulation Platforms}

A comprehensive feature-by-feature analysis of alternative simulation platforms is beyond the scope of this paper. Listed below are brief descriptions of some simulation systems using procedural methods, as opposed to JSim declarative approach. All can be used to fit model solutions to data. None provide automatic unit balance checking of equations

Virtual Cell (Loew \& Schaff, 2001) is a computational environment designed for the construction and simulation of cellular-based models. Models can be created iteratively in the GUI, or via VCell's custom mathematical language VCMDL which supports ODEs \& PDEs. Both deterministic and stochastic simulations are supported. Model computations are performed via client accounts on VCell's computational server farm. http://www.nrcam.uchc.edu/.

COPASI (Hoops et al., 2006) (for COmplex PAthway SImulator) is an integrated modeling and simulation environment aimed at metabolic networks, cell-signaling pathways, regulatory networks, infectious diseases and similar systems. Models are typically created via a table-driven GUI and results viewed via embedded graphs. COPASI supports SBML and currently runs on Linux, MacOS and Windows. http://www.copasi.org.

libRoadRunner (Sauro et al., 2013) is an high-performance simulation library supporting most SBML models: ODEs and events are supported, but algebraic rules are not. libRoadRunner provides APIs for $\mathrm{C}, \mathrm{C}++$ and Python to control model simulations. http:// libroadrunner.org/.

Chaste (Mirams et al., 2013) is C++ library for computational physiology and biology. Computational modules include mesh generation, linear algebra, ODEs, PDEs and continuum mechanics. I/O modules provide support for various file formats, including HDF5 (Folk et al., 1999). Chaste is available for Windows, MacOS, Linux and Solaris. https://chaste.cs.ox.ac.uk/.

PCenv, COR, OpenCell and OpenCOR (CellML, 2014) are a related set of tools supporting CellML modeling. PCenv is an interactive modeling editing and simulation environment running on Windows. COR an alternative CellML modeling environment for Windows. OpenCell is a merger of PCenv and COR built upon the Mozilla platform, and running on Linux, Windows and MacOS. OpenCell development has been stopped in favor of its replacement OpenCOR. http://www.cellml.org/tools.

Continuity (Continuity, 2014) is problem-solving environment for multi-scale modeling in bioengineering and physiology - especially biomechanics, transport and electrophysiology. Finite element and PDEs are supported. The Continuity language integrates with Python (VanRossum \& Drake, 2003) scripts to create multi-scale models. Continuity runs on Windows, MacOS, Linux and Linux clusters. http://cmrg.ucsd.edu/Continuity.

\section{Summary}

JSim is a tool for hypothesis exploration and for analyzing data. Many of the steps in data analysis are built into JSim. It's declarative modeling language, automatic unit balance checking, and builtin tools for solving ODEs, PDEs, and implicit equations greatly facilitate generating mathematically and physiologically consistent models. The built-in optimizers and associated statistical data reporting, along with behavior tools, such as parameter looping and 
sensitivity analysis, allow one to verify and explore model behavior in the context of experimental data and simulated data from previous models. With the ability to save these model 'explorations' as parameter sets within the JSim project file anyone can easily create a modeling and data analysis package that is easy to reproduce and distribute to others.

As a research tool, JSim has been developed and refined to accelerate the processes of modeling and data analysis. Adherence to quality standards augments efficiency (Smith et al., 2007). The time savings don't simply reduce the time necessary to get to a result, they also end up improving the quality of the science in two ways. First, when it only takes a few seconds to modify a model, re-run it, and view the results, researchers are more likely to explore many "what if" scenarios and develop a deeper understanding of model behavior, and in turn, a deeper understanding of the system being modeled. Second, researchers are more likely to do better verification checks and higher-level analyses if they are easy to do. When a few mouse clicks are all it takes to change solvers, time step sizes, optimization parameters, or even perform a complex Monte Carlo analysis to assess parameter correlations and confidence intervals, researchers are more likely to actually do those critical numerical checks and to take the model analysis beyond simply reporting a single parameter value.

In addition to its use as a research tool, JSim is also very useful as a teaching tool. JSim has been used in classes for high school, undergraduate, and graduate students, as well as many workshops for faculty members. The fact that JSim is open source, quick to download and install, as well as executable over the web, means that it is easily available to students. The simplicity of JSim's model specification language, where users can focus on writing and working with the mathematical equations themselves rather than controlling program flow, means that students with no programming experience can rapidly begin to understand, create, and modify JSim models. Furthermore, JSim's interactive plotting interface and the easy access it provides to sophisticated analysis tools such as sensitivity and Monte Carlo analysis allow students to perform analyses which would ordinarily be too difficult and time consuming for them to do on their own.

\section{Future developments:}

\section{Modular modeling}

JSim has provided support for modular modeling from its inception (Bassingthwaighte, 2000) using both mathematical and biological approaches, but now, with the developing recognition that models are more consistently understandable and more amenable to modular construction when they are annotated using identified ontology systems, libraries of modules present great opportunity for efficient construction of complex model systems. A module can be thought of as a self-contained model of an element of the larger system model and represents a specific physical, chemical or phenomenological process. A model might use multiple instances of the same module, for example, differently parameterized Michaelis-Menten type enzymatic reactions used for different reactants. One can build large models from a variety of modules representing physical or chemical processes such as the flux via a cell membrane transporter or ion channel or an enzymatic reaction, or a transcription regulatory pathway (Beard et al., 2005) incorporating knowledge of their connectivities. Allowing the modeler to draw pre-existing modules from a repository or extract them from previously developed models and enables the modeler to create new models quickly for hypothesis testing, a key to Physiome development (Bassingthwaighte et al., 2009). Below are two approaches to implementing modular modeling within JSim.

Modular Program Constructor (MPC): MPC focuses on using easily understood directives to extract generically coded JSim MML equations from files, changing the names of the generic variables to ontologically informative names and assembling the resulting code into new equations (Raymond \& Bassingthwaighte, 2011). For example, MPC can take MML code representing a single tissue exchange region (26 lines), and generate a whole organ heterogeneous model for convection, diffusion, and reaction with 20 regions (1698 lines). See http://www.physiome.org/jsim/models/webmodel/NSR/MPC/. MPC currently runs outside of JSim but is planned for incorporation into a future JSim release.

Modular construction with SemSim: Precise semantic identification of variables and parameters is a prerequisite to merging of preconstructed submodels or modules into integrated systems or multiscale models. A future release of JSim will incorporate the tools for annotating models and their computational elements against biomedical ontologies and knowledge bases (Rubin et al., 2006). These annotations will make it easier for users to search the Physiome Model repository and to identify the biological phenomena modeled. Formatted according to the semantic simulation (SemSim) framework (Gennari et al., 2011), these annotations will also make it possible for tools to decompose and merge models in a more automated fashion, and allow the modeler to work at a biological, rather than computational level of abstraction (Beard et al., 2012). For example, selection of an ion pump, such as the NaKATPase, would bring up a set of modules from which the modeler would choose the version suited to the particular context, and then the code for the integrated model would be automatically generated from the annotated modules in the library.

SED-ML Support: SED-ML (Kohn \& Le Novere, 2008) (for Simulation Experiment Description) is an emerging standard for to promote reproducibility by capturing all the details of an in silico experiment. Major entities described in SED-ML are models, simulation setup (i.e. time and numeric solver parameters), tasks (a model run with specified a simulation setup), data generators (methods for combining model outputs from different tasks) and outputs (plots \& tables). JSim projects support these entities, but not in a scripted form. To support SED-ML, we are currently developing a feature called Second Level Analysis (SLA) that will allow JSim users to script common JSim activities (e.g. model runs with different parameter sets, data combinatorics, plotting and export), in a way that maps to SED-ML. SED-ML files will be read into JSim as SLA scripts and run there. Conversely, JSim SLA scripts may be exported to SED-ML for use in other simulators that support SED-ML. 
Getting started with JSim:

Information for download and installation, running JSim, and writing JSim MML models can be found at http://www.physiome.org/jsim/.

\section{Data and software availability}

Zenodo: JSim downloads and models Version 2, doi: 10.5281/zenodo.8652 (Butterworth et al., 2014)

\section{Author contributions}

All authors contributed to the design and organization of the paper and its writing and editing. EB was the developer of the JSim engine and GUI. GR implemented numerical methods for numerical solvers and optimizers. BJ worked on the website and model coding, formatting and installation. MN developed large systems models and the methods of ontology annotation. JB was the overall systems designer and composed the integrated manuscript.

\section{Competing interests}

No competing interests were disclosed.

\section{Grant information}

The development of JSim has been supported by NIH grants HL9719 (PI: JBB), RR1243 (JBB), EB1273 (JBB), HL073598 (PI: R. Corley), EB8407 (JBB), and GM094503 (PI: D. Beard), GM081070 (PI: H. Sauro) and NSF grant 0506477 (JBB).

The funders had no role in study design, data collection and analysis, decision to publish, or preparation of the manuscript.

\section{Acknowledgements}

The authors would like to thank M. Bindschadler and H. Sauro for helpful discussions and feedback regarding the manuscript. Tom J. Knopp, Dennis U. Anderson, and Richard B. King contributed to the simulation methods used in the preceding simulation systems, SIMCON and XSIM, that were incorporated into JSim.
Aho A, Sethi R, Ullman J: Compilers: Principles, Techniques and Tools. AddisonWesley. 1988

Reference Source

Appel AW: Modern Compiler Implementation in Java. Cambridge University Press. 1998.

Reference Source

Barta E, Sideman S, Bassingthwaighte JB: Facilitated diffusion and membrane permeation of fatty acid in albumin solutions. Ann Biomed Eng. 2000; 28(3): 331-345.

PubMed Abstract | Publisher Full Text | Free Full Text

Bassingthwaighte JB: A concurrent flow model for extraction during

transcapillary passage. Circ Res. 1974; 35(3): 483-503.

PubMed Abstract | Publisher Full Text | Free Full Text

Bassingthwaighte JB, Goresky CA: Modeling in the analysis of solute and wate exchange in the microvasculature. In: Handbook of Physiology. Sect. 2, The Cardiovascular System. Vol IV The Microcirculation, edited by Renkin EM and Michel CC. Bethesda, MD: Am Physiol Soc. 1984; 549-626.

Bassingthwaighte JB, Chan IS, Goldstein AA, et al:: GGOPT: an unconstrained non-linear optimizer. Comput Methods Programs Biomed. 1988; 26(3): 275-81. PubMed Abstract | Publisher Full Text | Free Full Text

Bassingthwaighte JB, Wang $\mathrm{CY}$, Chan IS: Blood-tissue exchange via transport and transformation by capillary endothelial cells. Circ Res. 1989; 65(4): 997-1020.

PubMed Abstract | Publisher Full Text | Free Full Text

Bassingthwaighte JB, Chan IS, Wang CY: Computationally efficient algorithms for convection-permeation-diffusion models for blood-tissue exchange. Ann Biomed Eng. 1992; 20(6): 687-725.

PubMed Abstract

Bassingthwaighte JB: Strategies for the Physiome Project. Ann Biomed Eng. 2000; 28(8): 1043-58.

PubMed Abstract | Publisher Full Text | Free Full Text

Bassingthwaighte JB, Chizeck HJ, Atlas LE, et al.: Multiscale modeling of cardiac cellular Energetics. In: The Communicative Cardiac Cell. edited by Sideman S,

Beyar R and Landesberg A. Ann N Y Acad Sci. 2005; 1047: 395-424.

PubMed Abstract | Publisher Full Text | Free Full Text

Bassingthwaighte JB, Chizeck HJ, Atlas LE: Strategies and tactics in multiscale modeling of cell-to-organ systems. Proc IEEE Inst Electr Electron Eng. 2006; 94(4): 819-830.

PubMed Abstract | Publisher Full Text | Free Full Text

Bassingthwaighte JB, Raymond GR, Ploger JD, et al.: GENTEX, a general multiscale model for in vivo tissue exchanges and intraorgan metabolism.

Philos Trans A Math Phys Eng Sci. 2006; 364(1843): 1423-1442.

PubMed Abstract | Publisher Full Text

Bassingthwaighte J, Hunter P, Noble D: The Cardiac Physiome: perspectives fo the future. Exp Physiol. 2009; 94(5): 597-605.

PubMed Abstract | Publisher Full Text | Free Full Text

Bassingthwaighte JB: Modeling biological systems for reproducibility and sharing. TOPETJ (The Open Pacing, Electrophysiology, and Therapy Journal). 2010; 3: 66-74.

Bassingthwaighte JB, Chinn TM: Reexamining Michaelis-Menten enzyme kinetics for xanthine oxidase. Adv Physiol Educ. 2013; 37(1): 37-48. PubMed Abstract | Publisher Full Text | Free Full Text

Beard DA, Bassingthwaighte JB: The fractal nature of myocardial blood flow emerges from a whole-organ model of arterial network. J Vasc Res. 2000; 37(4): 282-96.

PubMed Abstract | Publisher Full Tex

Beard DA, Bassingthwaighte JB, Greene AS: Computational modeling of physiological systems. Physiol Genomics. 2005; 23(1): 1-3.

PubMed Abstract | Publisher Full Text

Beard DA, Neal ML, Tabesh-Saleki N, et al:: Multiscale modeling and data integration in the virtual physiological rat project. Ann Biomed Eng. 2012 40(11): 2365-78.

PubMed Abstract | Publisher Full Text | Free Full Text

Blom JG, Zegeling RUU: Algorithm 731: A moving-grid interface for systems of one-dimensional time-dependent partial differential equations. $A C M$

Transactions on Mathematical Software (TOMS). 1994; 2(2): 194-214.

Publisher Full Text

Butterworth E, Jardine BE, Raymond GM, et al.: JSim downloads and models Version 2. ZENODO 2014

Data Source

CellML tools. http://www.cellml.org/tools.

Chan IS, Goldstein AA, Bassingthwaighte JB: SENSOP: a derivative-free solver for non-linear least squares with sensitivity scaling. Ann. Biomed. Eng. 1993; 21(6): 621-31.

PubMed Abstract | Publisher Full Text | Free Full Text

Chizeck HJ, Butterworth E, Bassingthwaighte JB: Error detection and unit conversion. Automated unit balancing in modeling interface systems. IEEE Eng Med Biol. 2009; 28(3): 50-58.

PubMed Abstract | Publisher Full Text | Free Full Text

Coatrieux JL, Bassingthwaighte JB: Special Issue on the Physiome and Beyond. Proc IEEE. 2006; 94: 671-677.

Reference Source

Cohen SD, Hindmarsh AC: CVODE, a stiff/nonstiff solver in C 1995, UCRLJC-121014, (Cohen et al. ver. 2.4 Jul 2002).

Reference Source

Continuity 6: A Problem Solving Environment for Multi-Scale Biology. 2014 http://cmrg.ucsd.edu/Continuity

Cuellar AA, Lloyd CM, Nielsen PF, et al:: An Overview of CellML 1.1, a Biological Model Description Language. SIMULATION. 2003; 79(12): 740-747.

Publisher Full Text

Dantzig GB, Orden A, Wolfe P: The generalized simplex method for minimizing a linear form under linear inequality restraints. Pacific J Math. 1955; 5(2): 183-195. Reference Source

Dennis JE, Gay DM, Welsch RE: NL2SOL: An adaptive nonlinear least-squares 
algorithm. ACM Trans Math Softw. 1981; 7(3): 348-368.

Publisher Full Text

Dennis JE, Schnabel RB: Numerical methods for unconstrained optimization and nonlinear equation. N. Y.: Prentice-Hall, 1983; 378.

Reference Source

Escribano J, Garcia-Canovas F, Garcia-Carmona F: A kinetic study of

hypoxanthine oxidation by milk xanthine oxidase. Biochem J. 1988; 254(3): $829-33$

PubMed Abstract | Free Full Text

Euler L: Institutionum calculi integralis. 1768-1770.

Reference Source

Fehlberg E: Low-order classical Runge-Kutta formulas with stepsize control and their application to some heat transfer problems. NASA Technical Report-315. 1969.

Reference Source

Folk M, Cheng A, Yates K: HDF5: A file format and I/O library for high performance computing applications. Proceedings of Supercomputing. 99: 1999.

Gennari JH, Neal ML, Galdzicki M, et al.: Multiple ontologies in action: Composite annotations for biosimulation models. J Biomed Inform. 2011; 44(1): 146-154.

PubMed Abstract | Publisher Full Text | Free Full Text

Gillespie DT: Exact stochastic simulation of coupled chemical reactions. J Phys Chem. 1977; 81(25): 2340-2361.

Publisher Full Text

Goresky CA: A linear method for determining liver sinusoidal and extravascular volumes. Am J Physiol. 1963; 204: 626-640.

PubMed Abstract

Gosling J, McGilton H: The Java language environment: A white paper, 1996. Sun Microsystems. 2003; 85

Reference Source

Hairer E, Norsett SP, Wanner G: Solving Ordinary Differential Equations.

Nonstiff Problems. 2nd edition. Springer Series in Comput. Math., 1993; 8: 528.

Reference Source

Hairer E, Wanner G: Solving Ordinary Differential Equations. Stiff and

Differential-Algebraic Problems. 2nd edition. Springer Series in Comput. Math.,

1996; 14: 614

Reference Source

Harold ER: Java Network Programming. O’Reilly. 1997.

Reference Source

Harris PA, Bosan S, Harris TR, et al.: Parameter identification in coronary pressure flow models: a graphical approach. Ann Biomed Eng. 1994; 22(6) 622-637.

PubMed Abstract

Holland $\mathrm{JH}$ : Adaptation in natural and artificial Systems: an introductory analysis with applications to biology, control, and artificial intelligence. MIT Press. 1992; 183.

Reference Source

Hoops S, Sahle S, Gauges R, et al:: COPASI--a COmplex PAthway SImulator.

Bioinformatics. 2006; 22(24): 3067-74

PubMed Abstract | Publisher Full Text

Hucka M, Finney A, Sauro HM, et al:: SBML Forum. The systems biology markup language (SBML): a medium for representation and exchange of biochemical network models. Bioinformatics. 2003; 19(4): 524-31.

PubMed Abstract | Publisher Full Text

Jardine B, Bassingthwaighte JB: Modeling serotonin uptake in the lung shows endothelial transporters dominate over cleft permeation. Am J Physiol Lung Cell Mol Physiol. 2013; 305(1): L42-L55.

PubMed Abstract | Publisher Full Text | Free Full Text

King RB, Butterworth EA, Weissman LJ, et al.: A graphical user interface for computer simulation. FASEB J. 1995; 9: A14. (XSim).

Kirkpatrick S, Gelatt CD Jr, Vecchi MP: Optimization by simulated annealing. Science. 1983; 220(4598): 671-680.

PubMed Abstract | Publisher Full Text

Knopp TJ, Anderson DU, Bassingthwaighte JB: SIMCON--Simulation control to optimize man-machine interaction. Simulation. 1970; 14(2): 81-86.

PubMed Abstract | Publisher Full Text | Free Full Text

Kohn D, Le Novere N: SED-ML: an XML format for the implementation of the MIASE guidelines. In Computational Methods in Systems Biology. 2008; 176-190. Publisher Full Text

Kolda TG, Lewis RM, Torczon V: Optimization by direct search: New

perspectives on some classical and modern methods. Siam Review. 2003;

45(3): 385-482.

Publisher Full Text

Kuikka J, Levin M, Bassingthwaighte JB: Multiple tracer dilution estimates of D- and 2-deoxy-D-glucose uptake by the heart. Am J Physiol. 1986; 250(1 Pt 2): $\mathrm{H} 29-\mathrm{H} 42$.

PubMed Abstract | Free Full Text

LeVeque RJ: Finite Difference Methods for Ordinary and Partial Differential

Equations. Philadelphia: Siam. 2007.

Publisher Full Text

Liang S: The Java Native Interface: Programmer's Guide and Specification.
Addison-Wesley. 1999; 303

Reference Source

Loew L, Schaff J: The Virtual Cell: a software environment for computational cell biology. Trends Biotechnol. 2001; 19(10) : 401-6.

PubMed Abstract | Publisher Full Text

MacCormack RW: The Effect of viscosity in hypervelocity impact cratering.

AIAA Paper. 1969; 40(5): 69-354.

Publisher Full Text

Merson RH: An operational method for the study of integration processes.

Proc. Symp. Data Processing, Weapons Res. Establ. Salisbury, Salisbury. 1957; pp. 110-125.

Mirams GR, Arthurs CJ, Bernabeu MO, et al.: Chaste: an open source $\mathrm{C}_{++}$library for computational physiology and biology. PLoS Comput Biol. 2013; 9(3): e1002970.

PubMed Abstract | Publisher Full Text | Free Full Text

Neal ML, Bassingthwaighte JB: Subject-specific model estimation of cardiac output and blood volume during hemorrhage. Cardiovasc Eng. 2007; 7(3): 97-120.

PubMed Abstract | Publisher Full Text | Free Full Text

Nelder JA, Mead R: A simplex method for function minimization. Computer Journal. 1965; 7(4): 308-313.

Publisher Full Text

Oaks S, Wong H: Java Threads (Third Edition). O'Reilly. 2004.

Reference Source

Platt JR: Strong inference: Certain systematic methods of scientific thinking may produce much more rapid progress than others. Science. 1964; 146(3642): 347-353.

PubMed Abstract | Publisher Full Text

Poulain CA, Finlayson BA, Bassingthwaighte JB: Efficient numerical methods for nonlinear-facilitated transport and exchange in a blood-tissue exchange unit. Ann Biomed Eng. 1997; 25(3): 547-64.

PubMed Abstract | Publisher Full Text | Free Full Text

Raymond GM, Butterworth E, Bassingthwaighte JB: JSIM: Free software package for teaching physiological modeling and research. Exper Biol. 2003; 280(5): 102.

Raymond GM, Bassingthwaighte JB: Automated modular model construction using JSim. Experimental Biology. 2011; 863(9)

Reference Source

Roberts MS, Rowland M: A dispersion model of hepatic elimination: 1.

Formulation of the model and bolus considerations. J Pharmacokinet Biopharm. 1986; 14(3): 227-260.

PubMed Abstract

Rubin DR, Grossman D, Neal ML, et al: Ontology-based representation of simulation models of physiology. AMIA Annu Symp Proc. 2006; 664-668. PubMed Abstract | Free Full Text

Safford RE, Bassingthwaighte JB: Calcium diffusion in transient and steady states in muscle. Biophys J. 1977; 20(1): 113-136.

PubMed Abstract | Publisher Full Text | Free Full Text

Sauro H, Karlsson T, Swat M, et al:: libRoadRunner: A High Performance SBML Compliant Simulator. bioRxiv. 2013.

Publisher Full Text

Smith L, Butterworth E, Bassingthwaighte JB, et al.: SBML and CelIML translation in Antimony and JSim. (In press) Bioinformatics. 2013.

PubMed Abstract | Publisher Full Text | Free Full Text

Smith NA, Crampin EJ, Niederer SA, et al: Computational biology of the cardiac myocyte: proposed standards for the physiome. J Exper Biol. 2007; 210(Pt 9): 1576-1583.

PubMed Abstract | Publisher Full Text | Free Full Text

Smith R: Uncertainty Quantification: Theory, Implementation, and Applications. New York: SIAM Press. 2014; 384

Reference Source

Stone R, Jasny B: Scientific Discourse: Buckling at the Seams. Science. 2013; 342 (6154): 57-82. (Eight articles covering new views on improving scientific communication and accelerating research through openness and clarity.) Publisher Full Text

Suenson M, Richmond DR, Bassingthwaighte JB: Diffusion of sucrose, sodium, and water in ventricular myocardium. Am J Physiol. 1974; 227(5): 1116-1123. PubMed Abstract | Free Full Text

Van Rossum G, Drake FL: Python language reference manual. Network Theory. 2003. Reference Source

Vinnakota KC, Bassingthwaighte JB: Myocardial density and composition: a basis for calculating intracellular metabolite concentrations. Am J Physiol Heart Circ Physiol. 2004; 286(5): H1742-H1749.

PubMed Abstract | Publisher Full Tex

Winslow RL, Rice J, Jafri S, et al:: Mechanisms of altered excitation-contraction coupling in canine tachycardia-induced heart failure, II: Model studies. Circ Res. 1999; 84: 571-586.

PubMed Abstract | Publisher Full Text

Yngve G, Brinkley JF, Cook D, et al:: A model browser for biosimulation. AMIA Annu Symp Proc. 2007; 2007: 836-840.

PubMed Abstract | Free Full Text 


\section{Open Peer Review}

\section{Current Peer Review Status:}

\section{Version 2}

Reviewer Report 27 May 2014

https://doi.org/10.5256/f1000research.4229.r4743

(c) 2014 Nickerson D. This is an open access peer review report distributed under the terms of the Creative Commons Attribution License, which permits unrestricted use, distribution, and reproduction in any medium, provided the original work is properly cited.

\section{David Nickerson}

Auckland Bioengineering Institute, University of Auckland, Auckland, New Zealand

This revision of the manuscript and the discussion provided in the responses to reviewers improves the original manuscript. There are just a few very minor typographical errors that could be tidied up in any future revision of the manuscript.

\section{Section: "Run-time performance"}

Second paragraph, "....on the value of the variable themselves," should be "...on the value of the variables themselves,"

Table 4 title, "JSim Optimizers" should probably be "JSim Solvers"

\section{Section: "Some Alternative Simulation Platforms"}

Section title should follow the same case as other sections (i.e., sentence case).

First paragraph, "..as opposed to JSim declarative approach." should be "..as opposed to JSim's declarative approach."

First paragraph, missing final period at the end of the paragraph.

\section{Section: "SED-ML Support"}

Section title should also be sentence case.

"..[E]merging standard for to promote reproducibility.." - should probably delete the "for". In the responses to the reviewers comments, there is a bit of confusion on the relationship between FieldML and CellML. It is important, I think, to note that FieldML is completely independent of CelIML and, for example, will not be part of a CellML translator.

Competing Interests: No competing interests were disclosed.

I confirm that I have read this submission and believe that I have an appropriate level of 
expertise to confirm that it is of an acceptable scientific standard.

\section{Version 1}

Reviewer Report 23 January 2014

https://doi.org/10.5256/f1000research.3152.r2922

(C) 2014 Nickerson D. This is an open access peer review report distributed under the terms of the Creative Commons Attribution License, which permits unrestricted use, distribution, and reproduction in any medium, provided the original work is properly cited.

\section{David Nickerson}

Auckland Bioengineering Institute, University of Auckland, Auckland, New Zealand

This manuscript provides an introduction to, and description of, the JSim modelling system. The authors highlight the general purpose utility of this platform through the use of specific application examples, that are easily understood and followed by the reader and potential JSim users. This manuscript provides all appropriate links and examples that readers would require to get up and running with the JSim software.

This paper is well written, with just a few points that the authors may want to consider in future revisions of the manuscript.

The authors touch briefly on reuse of existing models/projects and the use of a CVS repository to archive the history of model development, as well as the discussion on modular modelling in the future developments section. The basis for this modularity and reuse seems to result in the development of a new, monolithic MML document for the assembled model. It might be useful to see if there are features in either JSim or MML that allow dynamic links to the source modules to be maintained allowing users to alternate sources or versions of the source modules (rather than the cut-and-paste style described in the manuscript).

In addition to the versioning of the JSim input data (experimental data, MML, projects, etc.), it is often the case that a specific piece of work requires some minimum version of the software itself. I wonder if there is any link between project files and JSim releases? For example, are users browsing the Physiome Repository able to determine if they need to update their version of JSim prior to loading a project file (or if in fact the software handles this internally).

The manuscript would benefit from a more thorough comparison of JSim to alternative tools, or at least some links to specific tools being contrasted in the article.

There is no description of how spatial geometries (finite element meshes or finite difference grids) are defined in JSim. Are the evolving standards for such descriptions (e.g., FieldML or SBML-spatial) being used or are there plans to use such? A comparison with approaches taken by tools like the Virtual Cell or Chaste might be useful. 
The authors make no reference to the adoption or interchange with the SED-ML standard. It would be useful to discuss any plans in this regard. Similarly, the evolving COMBINE archive format has a large overlap in aims with the JSim project file and the authors might want to comment on any plans to make use of that archiving format or contributions in that direction.

In some parts of the manuscript (e.g., the caption for figure 3) the description of the modelling/simulation example seems a bit excessive, and detracts from the primary focus of the article.

\section{Minor comments}

Page 5, column 1, first paragraph: Antimony is mentioned as a model import source format, but that format is not defined previously.

Page 10, column 1, third paragraph: "...using several different numerical method," missing 's' on method.

Competing Interests: No competing interests were disclosed.

\section{I confirm that I have read this submission and believe that I have an appropriate level of expertise to confirm that it is of an acceptable scientific standard.}

Author Response 17 Apr 2014

Erik Butterworth, University of Washington, Seattle, USA

"The authors touch briefly on reuse of existing models/projects and the use of a CVS repository to archive the history of model development, as well as the discussion on modular modelling in the future developments section. The basis for this modularity and reuse seems to result in the development of a new, monolithic $M M L$ document for the assembled model. It might be useful to see if there are features in either JSim or MML that allow dynamic links to the source modules to be maintained allowing users to alternate sources or versions of the source modules (rather than the cut-and-paste style described in the manuscript)."

We agree that JSim and MML modularity could be improved. We are currently in the design stages of a more modular form of MML that moves away from the current monolithic approach. Design goals for the new MML include the following: the ability to draw upon and reuse code modules stored either in a project file or in a permanent archived location on-line; support for the recently developed SBML "Hierarchical Model Composition" package; support for support for CellML v1.1 modular structuring; support for structured multiple reuse on modules (e.g. support of multiple parallel pathways); support for run-time switching of alternative modules; compatibility with existing MML models. However, we feel this work is not yet far enough along for public presentation.

"In addition to the versioning of the JSim input data (experimental data, MML, projects, etc.), it is often the case that a specific piece of work requires some minimum version of the 
software itself. I wonder if there is any link between project files and JSim releases? For example, are users browsing the Physiome Repository able to determine if they need to update their version of JSim prior to loading a project file (or if in fact the software handles this internally)."

All NSR provided models are curated to work properly with the current version of JSim, which is the version run in live Web applets. Users may also download JSim project for use on their own workstation, which may have an older JSim version installed. The vast majority of the time, JSim runs correctly on projects created by different JSim versions. The only difference will be that, when running an older JSim version, the latest features will not be available. There are three primary reasons why this is the case. First, the basic functionality of JSim and project file structure has been stable for many years. Second, the XML structure of project files allows old versions of JSim to ignore features in newer project files. Third, a version number tag in each project file allows newer versions of JSim to migrate old projects to the new format.

Occasionally, an NSR modeler discovers a JSim bug that must be fixed for proper operation. Release of that model will be delayed until a new version of JSim is released. In that case, our best practices recommendation is to note the minimum JSim version required to run the model on the relevant web page. For published papers, where it is important to reproduce figures "warts and all", it is recommended that the papers list the version of JSim used to produce the results.

Based on your comment, we discussed the possibility of making the JSim project version for each model more visible on our site. The consensus was that, given the generally high level of compatibility, this information would be mostly misleading to users by assigning an undeserved level of attention to the version number.

"The manuscript would benefit from a more thorough comparison of JSim to alternative tools, or at least some links to specific tools being contrasted in the article."

We've added a new section "Some Alternative Simulation Platforms" (page 18) that briefly discusses some alternative simulation platforms to JSim. Given the large number of available products (over 250 SBML-based products alone) the list is not exhaustive. Platforms mentioned are Virtual Cell, COPASI, roadRunner, Chaste, PCenv, COR, OpenCell, OpenCOR and Continuity. Given limited space, the descriptions of these programs are not complete nor is there a feature-to-feature comparison with JSim. Such a comparison, evaluating available mathematical methods, features, portability, usability, performance and scientific reproducibility would be a good idea for a follow-on paper to this one.

"There is no description of how spatial geometries (finite element meshes or finite difference grids) are defined in JSim. Are the evolving standards for such descriptions (e.g., FieldML or SBML-spatial) being used or are there plans to use such? A comparison with 
approaches taken by tools like the Virtual Cell or Chaste might be useful."

In $\mathrm{MML}$, at present, spatial grids are defined in regular $\mathrm{N}$-space. The $2 \mathrm{D}$ and $3 \mathrm{D}$ geometries used in finite element methods and higher dimensional PDEs are often specified using much more complex data structures. We forsee adding support for 2D and 3D PDEs on regularly spaced grids to JSim at some point in the not-too-distant future (JSim's MML compiler already supports parsing 2D and 3D PDEs; what is currently missing is appropriate numeric libraries). However, support for irregular high-dimension grids would require major changes to JSim and is not envisioned in the near future. We have monitored the standardization efforts of the SBML spatial extension and FieldML. At the point when any serious body of models exists for either of these formats, we will consider adding support for them to JSim's existing SBML and CellML translators. The utility of this effort will depend upon how many models in that body are runnable given JSim's limitation to regularly spaced grids.

"The authors make no reference to the adoption or interchange with the SED-ML standard. It would be useful to discuss any plans in this regard. Similarly, the evolving COMBINE archive format has a large overlap in aims with the JSim project file and the authors might want to comment on any plans to make use of that archiving format or contributions in that direction."

SED-ML support within JSim is currently under development. It is described in a section entitled "SED-ML Support" to the "Future Developments" section (page 20) of the paper to describe our plans there.

COMBINE looks like an interesting project, albeit one in the preliminary stages. But why would it be advantageous to migrate away from JSim project files? Should COMBINE become widely adopted, we will certainly consider supporting COMBINE import and export options within JSim. I think COMBINE is too preliminary for mention in the paper, however.

"In some parts of the manuscript (e.g., the caption for figure 3) the description of the modelling/simulation example seems a bit excessive, and detracts from the primary focus of the article."

Figure 3 is problem, we agree. But it takes quite a description to convey understudying of what can be rapidly learned from such multidimensional plots. Complex, but easy to produce and rapid to interpret after learning how. We think the legend is needed so that a reader can determine how valuable the feature might be, even though it takes space.

\section{Minor comments:}

"Page 5, column 1, first paragraph: Antimony is mentioned as a model import source format, but that format is not defined previously."

"Page 10, column 1, third paragraph: "...using several different numerical method," missing 's' on method."

Thank you. Text now corrected. 
Competing Interests: None.

Reviewer Report 10 January 2014

https://doi.org/10.5256/f1000research.3152.r2924

(C) 2014 Niederer S. This is an open access peer review report distributed under the terms of the Creative Commons Attribution License, which permits unrestricted use, distribution, and reproduction in any medium, provided the original work is properly cited.

\section{Steven Niederer}

Biomedical Engineering Department, King's College London, London, UK

The paper "JSim, an open-source modelling system for data analysis" provides a succinct update on the functionality and utility of the modelling platform JSim. The paper provides a concise description and link between the JSim community modelling philosophy and how this is facilitated by the JSim software platform. The complete description of the JSim environment will be of interest to the modelling community and this manuscript highlights much of the functionality that they require.

This publication could be improved by addressing:

As described in the article, the platform JSim has been developed over a period of over 40 years. Previous articles on JSim have been published and this article would be strengthened by highlighting the new features / functionality added to the platform since the previous JSim article.

The article, as exemplified in the abstract, focuses on the technical functionality of JSim. If JSim can be readily set up or used by people who wish to analyse their experimental data with models, for example experimental researchers, as opposed to developing new models, then it would be worth highlighting this in the abstract and text.

The article does not discuss or review alternate simulation platforms (for example COR, OpenCell, Continuity, CHASTE, SBML simulation environments). For new users wishing to make an informed decision it would be useful to highlight the differences between JSim and alternate platforms.

\section{Minor comments}

In the introduction, the statement that mathematical models provide clear and precise hypothesis that are susceptible to contradiction and that failure to fit leads to rejection needs to be more nuanced, particularly in the case of biology where comparisons are often made between deterministic models and variable experimental results.

It is not clear in the loop section if JSim supports nested loops, this could be clarified. 
It would be of interest to provide some indicative performance measures. For example if simulating a cardiac action potential will JSim solve faster or slower than real time on a conventional desktop.

The authors could comment on the utility or potential for adoption of new mark-up languages for spatial problems (FieldML) or problem definition formats (SED-ML).

It would be interesting for the authors to comment on how or if they have verified the JSim code stack.

Competing Interests: No competing interests were disclosed.

\section{I confirm that I have read this submission and believe that I have an appropriate level of expertise to confirm that it is of an acceptable scientific standard.}

Author Response 17 Apr 2014

Erik Butterworth, University of Washington, Seattle, USA

"As described in the article, the platform JSim has been developed over a period of over 40 years. Previous articles on JSim have been published and this article would be strengthened by highlighting the new features/functionality added to the platform since the previous JSim article."

This is the first "complete" of description of JSim to be published. Previous papers have made use of JSim as a tool, but did not describe the software or the systematic approach to it. A comparison of JSim functionality to its predecessors, SimCon and XSim, is in the section labeled "Background" in the paper's introduction.

"The article, as exemplified in the abstract, focuses on the technical functionality of JSim. If JSim can be readily set up or used by people who wish to analyse their experimental data with models, for example experimental researchers, as opposed to developing new models, then it would be worth highlighting this in the abstract and text."

Good suggestion. One sentence is added to the abstract, and four sentences added on page 16 in a new section just before the Summary entitled "Using archived models for analyzing one's own data"

"The article does not discuss or review alternate simulation platforms (for example COR, OpenCell, Continuity, CHASTE, SBML simulation environments). For new users wishing to make an informed decision it would be useful to highlight the differences between JSim and alternative platforms."

Although this paper's main focus is a fairly detailed description of JSim, we've added a new section "Some Alternative Simulation Platforms" (page 18) that discusses alternative simulation platforms to JSim. Given the large number of available products (over 250 SBML-based products alone) the list is not exhaustive. Platforms discussed are Virtual Cell, COPASI, roadRunner, Chaste, PCenv, COR, OpenCell, 
OpenCOR and Continuity. The descriptions of these programs are not complete and there are no feature-to-feature comparison between them and JSim. Detailed comparison, evaluating available mathematical methods, features, portability, usability, performance, and scientific reproducibility would be a good idea for a follow-on paper.

\section{Minor Comments:}

"In the introduction, the statement that mathematical models provide clear and precise hypothesis that are susceptible to contradiction and that failure to fit leads to rejection needs to be more nuanced, particularly in the case of biology where comparisons are often made between deterministic models and variable experimental results."

The second paragraph of the Introduction has been revised accordingly.

"It is not clear in the loop section if JSim supports nested loops, this could be clarified."

JSim currently supports 2 loop nesting levels (inner loops and outer loops). This has been clarified in the section "Loops: Iterating solutions to exhibit behaviour" (page 11).

"It would be of interest to provide some indicative performance measures. For example if simulating a cardiac action potential will JSim solve faster or slower than real time on a conventional desktop."

Since JSim is a general purpose modeling engine, it can't be said that there is a particular "standard" model upon which to base comparisons. JSim users typically write models that run somewhere between a small fraction of a second to several minutes depending upon the complexity and numeric methods required. In particular, spatial models using PDEs compute much slower than compartmental models using ODEs. Most pharmacokinetic and whole body circulatory models run faster than real time, but there are exceptions, e.g. models with multistage receptors, transporters and complicated reaction networks. Under Run Time Performance on p 15, we've added a description of ODE and PDE assessment of model solution times in millisecond solution times per second of model time, comparing solvers and ODEs versus PDEs. This new section gives an overview of the various factors affecting run-time performance, and gives some run times for a selected set of models (including a BR cardiac action potential model). These run times vary from about 13,966 times faster than real time to about 5 times slower.

We have a paper in preparation that discusses comparative performance in more detail, and will contrast JSim performance with several other computational engines for SBML and Matlab models.

"The authors could comment on the utility or potential for adoption of new mark-up languages for spatial problems (FieldML) or problem definition formats (SED-ML)."

SED-ML support within JSim is currently under development. We have added a section entitled "SED-ML Support" to the fact "Future Plans" (page 20) section of the paper to 
describe our plans there.

Support for FieldML is a more problematic. FieldML provides a mathematical framework for calculating a CellML point models over space, defined in terms of a real topological manifold. In the field of 2D and 3D computation, such manifold representations are often complex, irregular spatial grids. At present, JSim represents $\mathrm{N}$-dimensional space as a cross product of regularly spaced grids.

Support for generalized manifolds would be a major change in functionality requiring significant time and resources. While such a change might be contemplated some point in future, it is not within our immediate plans. JSim could support a limited version of FieldML, supporting the subset of real manifolds that are regular grids in $\mathrm{N}$-space, but it is unclear what would be gained from a user's point of view. The primary advantage of standard ML support is the ability to access existing archives and exchange models between modeling systems. Unlike CellML and SBML, there is no evidence of large archives of FieldML models that can be run on a variety of platforms; our understanding is that FieldML-defined model can be run only in CMiss at this point. It can be considered bothersome to have separate archival forms for PDEs and ODEs. What one wants for ease of reproducibility is one self-consistent system, preferably all in one file. JSim does this for a limited repertoire of ODEs, 1and 2-D PDEs, and DAEs.

"It would be interesting for the authors to comment on how or if they have verified the JSim code stack."

Thanks for this suggestion. We've added a new section "Code Verification" (page 16) to address this topic. In brief, we use a combination of various strategies including comparison with analytic solutions, test statistics, comparison with other computational environments and round-tripping. Bug reports from modelers who find computations that violate physical intuition play a major role. An automated verification suite of anointed regression tests ensures continued compliance over subsequent releases.

Competing Interests: None. 
The benefits of publishing with F1000Research:

- Your article is published within days, with no editorial bias

- You can publish traditional articles, null/negative results, case reports, data notes and more

- The peer review process is transparent and collaborative

- Your article is indexed in PubMed after passing peer review

- Dedicated customer support at every stage

For pre-submission enquiries, contact research@f1000.com 